BULLETIN (New Series) OF THE

AMERICAN MATHEMATICAL SOCIETY

Volume 42, Number 2, Pages 181-211

S 0273-0979(05)01050-5

Article electronically published on January 26, 2005

\title{
WHAT IS KNOWN ABOUT UNIT CUBES
}

\author{
CHUANMING ZONG
}

\begin{abstract}
Unit cubes, from any point of view, are among the simplest and the most important objects in $n$-dimensional Euclidean space. In fact, as one will see from this survey, they are not simple at all. On the one hand, the known results about them have been achieved by employing complicated machineries from Number Theory, Group Theory, Probability Theory, Matrix Theory, Hyperbolic Geometry, Combinatorics, etc.; on the other hand, the answers for many basic problems about them are still missing. In addition, the geometry of unit cubes does serve as a meeting point for several applied subjects such as Design Theory, Coding Theory, etc. The purpose of this article is to figure out what is known about the unit cubes and what do we want to know about them.
\end{abstract}

\section{INTRODUCTION}

Taking a unit box (a three-dimensional unit cube) in a hand, one can easily see that it is a very symmetric object with six faces, twelve edges and eight vertices. In addition, one can simply conclude that its volume is one and its surface area is six. Then a layman perhaps will have no further questions and is satisfied with the belief that he has known everything about the box. However, a geometer may ask further questions of the following types.

1. What is the maximum area of its cross sections?

2. What is the maximum area of its projections?

3. What is the maximum volume of a tetrahedron inscribed in the box?

4. What is the smallest number of simplices to triangulate the box?

In fact, they are nontrivial problems. Especially, their analogues in higher dimensions are important, fascinating and challenging.

Let $R$ denote the real number field, let $E^{n}$ denote the $n$-dimensional Euclidean space, let small boldface letters denote points (or vectors) in $E^{n}$ and let the corresponding small letters with lower indices denote their coordinates. Especially, the origin of $E^{n}$ is denoted by $\mathbf{o}$ in the whole paper. For different purposes, we define two particular unit cubes:

$$
I^{n}=\left\{\mathbf{x}=\left(x_{1}, x_{2}, \cdots, x_{n}\right) \in E^{n}:\left|x_{i}\right| \leq \frac{1}{2}\right\}
$$

Received by the editors June 9, 2004

2000 Mathematics Subject Classification. Primary 05A15, 05B20, 05B25, 05B45, 11H31, 11J13, 15A33, 20K01, 28A25, 52B05, 52C20, 52C22.

This work was supported by the National Science Foundation of China, 973 Project and a special grant from Peking University. 
and

$$
\overline{I^{n}}=\left\{\mathbf{x}=\left(x_{1}, x_{2}, \cdots, x_{n}\right) \in E^{n}: 0 \leq x_{i} \leq 1\right\} .
$$

To have some intuition about the geometric shape of an $n$-dimensional unit cube, one may define it inductively as a cylinder based on an $(n-1)$-dimensional one. In this way, one can deduce that an n-dimensional unit cube has exactly $2^{n-k}\left(\begin{array}{c}n \\ n-k\end{array}\right)$ different $k$-dimensional faces, each of which is a $k$-dimensional unit cube.

The geometry of unit cubes is a meeting point of several different subjects in mathematics. For example, as one will see in the following sections, Probability Theory does play an important role in the study of cross sections, Linear Algebra is fundamental in the study of both projections and inscribed simplices, Combinatorics is basic for both triangulations and 0/1 polytopes, and Group Theory is essential in the study of both Minkowski's conjecture and Keller's conjecture. In addition, Keller's conjecture, inscribed simplices, $0 / 1$ polytopes and triangulations are closely related with applied subjects such as Coding Theory and Design Theory.

In this article we will review several important topics about $n$-dimensional unit cubes, such as cross sections, projections, inscribed simplices, Minkowski's conjecture, triangulations, Keller's conjecture, etc. Besides introducing the fundamental results and some key open problems, we will briefly discuss some creative ideas by which the fascinating results have been achieved. For a detailed study we refer to the original papers or to Zong [117.

\section{Cross-Sections}

Problem 1.1. What is the maximum or minimum area of an $i$-dimensional crosssection of $I^{n}$ ?

This problem is so natural that it makes no sense to ask whoever first proposed it. However, it is indeed a challenging one. K. Ball, D. Hensley and J.D. Vaaler have made essential progress in this problem and have solved many particular cases. However, a complete solution is still missing. In addition, the proofs for the known results are based on deep and unexpectedly complicated analysis.

Let $H^{i}$ denote an $i$-dimensional hyperplane containing $\mathbf{o}$ and let $v_{i}(X)$ denote the $i$-dimensional measure of a set $X$ in $E^{n}$. According to Hensley 49], Anton Good made the following conjecture.

Good's conjecture. If $1 \leq i \leq n-1$, then

$$
v_{i}\left(I^{n} \cap H^{i}\right) \geq 1 \text {. }
$$

In 1979, unexpectedly, Hensley [49] introduced a probability method into the study of this conjecture and solved the $i=n-1$ case. Almost at the same time, J.D. Vaaler improved Hensley's method into a much more powerful setting and proved a fundamental theorem about section measure, by which one can deduce Good's conjecture as a corollary. Let $\overline{B^{j}}$ denote the $j$-dimensional ball of unit $j$ dimensional volume and centered at the origin of the space and let $\chi(V, \mathbf{x})$ denote the characteristic function of a set $V$. Then Vaaler's theorem can be stated as follows.

Theorem 1.1 (Vaaler [107). Suppose that $n_{1}, n_{2}, \cdots, n_{j}$ are positive integers satisfying $n=n_{1}+n_{2}+\cdots+n_{j}, D=\overline{B^{n_{1}}} \oplus \overline{B^{n_{2}}} \oplus \cdots \oplus \overline{B^{n_{j}}} \subset E^{n}$, and $A$ is an 
$i \times n$ real matrix of rank $i$. Then we have

$$
\int_{E^{i}} \chi(D, \mathbf{x} A) d \mathbf{x} \geq\left|A A^{\prime}\right|^{-\frac{1}{2}},
$$

where $A^{\prime}$ is the transpose of $A$.

Taking $n_{1}=n_{2}=\cdots=n_{n}=1$ and choosing $A$ such that its rows form an orthonormal basis for $H^{i}$ in $E^{n}$, then we have $D=I^{n},\left|A A^{\prime}\right|=1$ and

$$
\int_{E^{i}} \chi(D, \mathbf{x} A) d \mathbf{x}=v_{i}\left(I^{n} \cap H^{i}\right) .
$$

Thus, Good's conjecture follows as a corollary.

Corollary 1.1 (Vaaler [107]). If $1 \leq i \leq n-1$, then

$$
v_{i}\left(I^{n} \cap H^{i}\right) \geq 1 \text {. }
$$

In the proof of Vaaler's theorem, some deep analytic methods do play very important roles. Let us start with a couple of basic concepts. A nonnegative function $f(\mathbf{x})$ defined in $E^{n}$ is said to be logconcave if

$$
f\left(\lambda \mathbf{x}_{1}+(1-\lambda) \mathbf{x}_{2}\right) \geq f\left(\mathbf{x}_{1}\right)^{\lambda} f\left(\mathbf{x}_{2}\right)^{1-\lambda}
$$

holds for every pair of points $\mathbf{x}_{1}$ and $\mathbf{x}_{2}$ in $E^{n}$ and for every $\lambda$ with $0<\lambda<1$. Similarly, a probability measure $\mu$ defined on $E^{n}$ is said to be logconcave if

$$
\mu\left(\lambda K_{1}+(1-\lambda) K_{2}\right) \geq \mu\left(K_{1}\right)^{\lambda} \mu\left(K_{2}\right)^{1-\lambda}
$$

holds for every pair of open convex sets $K_{1}$ and $K_{2}$ in $E^{n}$ and for every $\lambda$ with $0<\lambda<1$. Logconcave functions and logconcave probability measures are closely related. It was shown by Borell [15] and Prékopa [88] that, roughly speaking, $\mu$ is a logconcave probability measure if and only if there is a logconcave function $f(\mathbf{x})$ defined on some $i$-dimensional subspace $H^{i}$ of $E^{n}$ such that

$$
d \mu=f(\mathbf{x}) d \nu_{i},
$$

where $\nu_{i}$ is the $i$-dimensional Lebesgue measure on $H^{i}$.

Let $\mu_{1}$ and $\mu_{2}$ be probability measures with density functions $f_{1}(\mathbf{x})$ and $f_{2}(\mathbf{x})$, respectively. We say that $\mu_{1}$ (or $\left.f_{1}(\mathbf{x})\right)$ is more peaked than $\mu_{2}$ (or $f_{2}(\mathbf{x})$ ) if

$$
\mu_{1}(C) \geq \mu_{2}(C)
$$

holds for every centrally symmetric convex body $C$ centered at o. It can be shown that both $\chi\left(\overline{B^{i}}, \mathbf{x}\right)$ and $e^{-\pi\|\mathbf{x}\|^{2}}$ are logconcave and $\chi\left(\overline{B^{i}}, \mathbf{x}\right)$ is more peaked than $e^{-\pi\|\mathbf{x}\|^{2}}$. In addition, one can prove (see Kanter [60]) that $\mu_{1} \otimes \mu_{2}$ is more peaked than $\mu_{1}^{\prime} \otimes \mu_{2}^{\prime}$ if $\mu_{1}, \mu_{2}, \mu_{1}^{\prime}$ and $\mu_{2}^{\prime}$ are logconcave, $\mu_{1}$ is more peaked than $\mu_{1}^{\prime}$ and $\mu_{2}$ is more peaked than $\mu_{2}^{\prime}$. Therefore $\chi(D, \mathbf{x})$ is more peaked than $e^{-\pi\|\mathbf{x}\|^{2}}$; that is,

$$
\int_{C} e^{-\pi\|\mathbf{x}\|^{2}} d \mathbf{x} \leq \int_{C} \chi(D, \mathbf{x}) d \mathbf{x}
$$

holds for every centrally symmetric convex body $C$ centered at $\mathbf{o}$.

Let $E^{i}$ denote the $i$-dimensional subspace of $E^{n}$ spanned by the rows of $A$, let $E^{n-i}$ denote its orthogonal complement, let $B$ denote an $(n-i) \times n$ matrix such that its rows form an orthonormal basis in $E^{n-i}$ and let $I^{n-i}$ denote a unit cube in $E^{n-i}$ and centered at its origin. Writing

$$
T=\left(\begin{array}{l}
A \\
B
\end{array}\right)
$$


and

$$
H_{\epsilon}=E^{i}+\epsilon I^{n-i},
$$

where + is the Minkowski sum and $\epsilon$ is a small positive number, by (1.1) we have

$$
\int_{H_{\epsilon}} e^{-\pi\|\mathbf{x} T\|^{2}} d \mathbf{x} \leq \int_{H_{\epsilon}} \chi(D, \mathbf{x} T) d \mathbf{x},
$$

by which one can deduce Theorem 1.1.

Slightly before Vaaler's work, Hensley [49] did prove

$$
1 \leq v_{n-1}\left(I^{n} \cap H^{n-1}\right) \leq 5
$$

and made a conjecture that

$$
v_{n-1}\left(I^{n} \cap H^{n-1}\right) \leq \sqrt{2} .
$$

As for a general upper bound for $v_{i}\left(I^{n} \cap H^{i}\right)$, K. Ball proved the following two theorems.

Theorem 1.2 (Ball [7]). For every $i$-dimensional hyperplane $H^{i}$ in $E^{n}$ we have

$$
v_{i}\left(I^{n} \cap H^{i}\right) \leq\left(\frac{n}{i}\right)^{\frac{i}{2}},
$$

where the upper bound is best possible if $i \mid n$.

Theorem 1.3 (Ball [7]). For every $i$-dimensional hyperplane $H^{i}$ in $E^{n}$ we have

$$
v_{i}\left(I^{n} \cap H^{i}\right) \leq 2^{\frac{n-i}{2}},
$$

where the upper bound is optimal if $i \geq n / 2$.

It is easy to see that these theorems do provide an answer to Problem 1.1 for many cases, especially to Hensley's conjecture. However, the answers to many other cases are still missing.

Ball's proofs were based on deep analysis of another character. Let $\mathbf{u}_{i}$ be $m$ unit vectors in $E^{n}$ and let $c_{i}$ be $m$ positive numbers $(m \geq n)$ satisfying

$$
\sum_{i=1}^{m} c_{i} \mathbf{u}_{i} \otimes \mathbf{u}_{i}=I_{n}
$$

where $\mathbf{u}_{i} \otimes \mathbf{u}_{i}$ indicates the tensor product and $I_{n}$ is the $n \times n$ unit matrix. Then for nonnegative integrable functions $f_{i}$ we have

$$
\int_{E^{n}} \prod_{i=1}^{m} f_{i}\left(\left\langle\mathbf{u}_{i}, \mathbf{x}\right\rangle\right)^{c_{i}} d \mathbf{x} \leq \prod_{i=1}^{m}\left(\int_{R} f_{i}(x) d x\right)^{c_{i}},
$$

where the equality holds if $f_{i}(x)$ are identical Gaussian densities. This is a special case of the Brascamp-Lieb inequality (see [17]).

Let $\mathbf{e}_{1}, \mathbf{e}_{2}, \cdots, \mathbf{e}_{n}$ be a standard basis of $E^{n}$ and let $\Gamma$ denote the orthogonal projection onto $H^{i}$. Taking

$$
c_{j}=\left\|\Gamma\left(\mathbf{e}_{j}\right)\right\|^{2} \quad \text { and } \quad \mathbf{u}_{j}=\frac{1}{\sqrt{c_{j}}} \Gamma\left(\mathbf{e}_{j}\right),
$$

one can deduce

$$
\sum_{j=1}^{n} c_{j} \mathbf{u}_{j} \otimes \mathbf{u}_{j}=I_{i} .
$$


On the other hand, letting $g_{j}(x)$ denote the characteristic function of the interval $\left[-\frac{1}{2 \sqrt{c_{j}}}, \frac{1}{2 \sqrt{c_{j}}}\right]$, it can be shown that

$$
v_{i}\left(I^{n} \cap H^{i}\right)=\int_{H^{i}} \prod_{j=1}^{n} g_{j}\left(\left\langle\mathbf{u}_{j}, \mathbf{x}\right\rangle\right)^{c_{j}} d \mathbf{x} .
$$

By (1.2) one can deduce Theorem 1.2.

To prove Theorem 1.3, besides the above method, Fourier's inversion formula plays an important role. Let $\overline{H^{i}}$ denote the orthogonal complement of $H^{i}$ and, for any $\mathbf{v} \in \overline{H^{i}}$, define

$$
f(\mathbf{v})=v_{i}\left(I^{i} \cap\left(H^{i}+\mathbf{v}\right)\right) .
$$

One can deduce

$$
\int_{\overline{H^{i}}} e^{i\langle\mathbf{v}, \mathbf{w}\rangle} f(\mathbf{v}) d \mathbf{v}=\prod_{j=1}^{n} \frac{\sin \frac{\sqrt{c_{j}}}{2}\left\langle\mathbf{w}, \mathbf{u}_{j}\right\rangle}{\frac{\sqrt{c_{j}}}{2}\left\langle\mathbf{w}, \mathbf{u}_{j}\right\rangle} .
$$

By the standard Fourier inversion formula we have

$$
v_{i}\left(I^{n} \cap H^{i}\right)=f(\mathbf{o})=\frac{1}{(2 \pi)^{n-i}} \int_{\overline{H^{i}}} \prod_{j=1}^{n} \frac{\sin \frac{\sqrt{c_{j}}}{2}\left\langle\mathbf{w}, \mathbf{u}_{j}\right\rangle}{\frac{\sqrt{c_{j}}}{2}}\left\langle\mathbf{w}, \mathbf{u}_{j}\right\rangle \text {. }
$$

Then Theorem 1.3 can be deduced by (1.2) and the fact that if $\lambda \geq 2$,

$$
\frac{1}{\pi} \int_{-\infty}^{\infty}\left|\frac{\sin t}{t}\right|^{\lambda} d t \leq \sqrt{\frac{2}{\lambda}}
$$

For convenience, let $\alpha(n, i)$ denote the maximum area of an $i$-dimensional cross section of $I^{n}$. By Theorem 1.2 and Theorem 1.3, most values of $\alpha(n, i)$ are known when $n$ is relatively small. We list them up to $n=12$ in Table 1 .

\section{TABLE 1.}

\begin{tabular}{|c|c|c|c|c|c|c|c|c|c|c|c|c|}
\hline$i$ & 1 & 2 & 3 & 4 & 5 & 6 & 7 & 8 & 9 & 10 & 11 & 12 \\
\hline$\alpha(3, i)$ & $\sqrt{3}$ & $\sqrt{2}$ & 1 & & & & & & & & & \\
\hline$\alpha(4, i)$ & 2 & 2 & $\sqrt{2}$ & 1 & & & & & & & & \\
\hline$\alpha(5, i)$ & $\sqrt{5}$ & $? ?$ & 2 & $\sqrt{2}$ & 1 & & & & & & & \\
\hline$\alpha(6, i)$ & $\sqrt{6}$ & 3 & $\sqrt{8}$ & 2 & $\sqrt{2}$ & 1 & & & & & & \\
\hline$\alpha(7, i)$ & $\sqrt{7}$ & $? ?$ & $? ?$ & $\sqrt{8}$ & 2 & $\sqrt{2}$ & 1 & & & & & \\
\hline$\alpha(8, i)$ & $\sqrt{8}$ & 4 & $? ?$ & 4 & $\sqrt{8}$ & 2 & $\sqrt{2}$ & 1 & & & & \\
\hline$\alpha(9, i)$ & 3 & $? ?$ & $\sqrt{27}$ & $? ?$ & 4 & $\sqrt{8}$ & 2 & $\sqrt{2}$ & 1 & & & \\
\hline$\alpha(10, i)$ & $\sqrt{10}$ & 5 & $? ?$ & $? ?$ & $\sqrt{32}$ & 4 & $\sqrt{8}$ & 2 & $\sqrt{2}$ & 1 & & \\
\hline$\alpha(11, i)$ & $\sqrt{11}$ & $? ?$ & $? ?$ & $? ?$ & $? ?$ & $\sqrt{32}$ & 4 & $\sqrt{8}$ & 2 & $\sqrt{2}$ & 1 & \\
\hline$\alpha(12, i)$ & $\sqrt{12}$ & 6 & 8 & 9 & $? ?$ & 8 & $\sqrt{32}$ & 4 & $\sqrt{8}$ & 2 & $\sqrt{2}$ & 1 \\
\hline
\end{tabular}

As for the shapes of the cross sections of $I^{n}$, our knowledge is very limited. According to a well-known theorem of Dvoretzky [28], for any fixed $k$, when $n$ 
is sufficiently large there is a $k$-dimensional hyperplane $H$ such that $I^{n} \cap H$ is almost spherical. On the other hand, any n-dimensional centrally symmetric convex polytope with $m$ pairs of facets can be realized as an $n$-dimensional cross section of an m-dimensional cube (see Ball [8]). In addition, according to Bárány and Lovász [9], if a $k$-dimensional cross section of $I^{n}$ has no common point with the $(n-k-1)$ dimensional faces of $I^{n}$, then it has at least $2^{k}$ vertices. However, we do not know any good bound for the number of the $j$-dimensional faces of a $k$-dimensional cross section of $I^{n}$. Let $E(n, k, j)$ denote the expected number of $j$-dimensional faces of a random $k$-dimensional cross section of $I^{n}$. Lonke 72 recently proved

$$
E(n, k, 0)=2^{k}\left(\begin{array}{l}
n \\
k
\end{array}\right) \sqrt{\frac{2 k}{\pi}} \int_{0}^{\infty} e^{-k t^{2} / 2} \mu_{n-k}\left(t I^{n-k}\right) d t
$$

where $\mu_{n-k}$ indicates the $(n-k)$-dimensional Gaussian probability measure, and

$$
E(n, n-k, n-j) \sim \frac{(2 n)^{j-k}}{(j-k) !}
$$

for fixed $1 \leq k<j$ as $n \rightarrow \infty$. As consequences of the first formula, for fixed $k$, when $n \rightarrow \infty$ one can deduce

$$
E(n, k, 0) \sim \frac{2^{k}}{\sqrt{k}}(\pi \log n)^{(k-1) / 2}
$$

and

$$
E(n, n-1,0) \sim \frac{2^{n} \sqrt{n}}{\pi}
$$

\section{Projections}

Similar to Problem 1.1, we have the following problem about the projections of $I^{n}$.

Problem 2.1. What is the maximum (minimum) area of an $i$-dimensional projection of $I^{n}$ ?

It is known (see Table 1) that the maximum area of a two-dimensional cross section of $I^{3}$ is $\sqrt{2}$. However, by routine computation one can deduce that the maximum area of a two-dimensional projection of $I^{3}$ is $\sqrt{3}$. This example does show the essential difference between cross sections and projections of $I^{n}$. In addition, as one will see, while the key method to deal with the cross sections is analytic the basic technique for projections is algebraic.

Let $H^{i}$ denote an $i$-dimensional hyperplane containing o and let $P_{i}$ denote the orthogonal projection from $I^{n}$ to $H^{i}$. It is easy to see that $P_{i}$ is a polytope and

$$
I^{n} \cap H^{i} \subseteq P_{i}
$$

holds for every $H^{i}$. Therefore, by Theorem 1.1 we have the following lower bound for $v_{i}\left(P_{i}\right)$.

Theorem 2.1 (Chakerian and Filliman [20]). If $1 \leq i \leq n-1$, for any $i$ dimensional orthogonal projection $P_{i}$ of $I^{n}$ we have

$$
v_{i}\left(P_{i}\right) \geq 1 \text {, }
$$

where the equality holds if and only if $H^{i}$ is spanned by $i$ axes of $E^{n}$. 
Turning to the upper bound, the situation is much more complicated. Let us start with some easy observations. It is obvious that $I^{n}$ is contained in a ball of radius $\sqrt{n} / 2$. Therefore, for any $i$-dimensional projection $P_{i}$ of $I^{n}$ we have

$$
v_{i}\left(P_{i}\right) \leq \omega_{i} \cdot(\sqrt{n} / 2)^{i}
$$

where $\omega_{i}$ is the volume of the $i$-dimensional unit ball.

Write $q=[n /(i+1)]$. For $j=1,2, \cdots, i+1$, let $\mathbf{p}_{j}=\left(p_{j 1}, p_{j 2}, \cdots, p_{j n}\right)$ be the vertex of $\overline{I^{n}}$ with coordinates

$$
p_{j k}= \begin{cases}1 & \text { if }(j-1) q+1 \leq k \leq j q, \\ 0 & \text { otherwise }\end{cases}
$$

It can be verified that the simplex $S$ with vertices $\mathbf{p}_{1}, \mathbf{p}_{2}, \cdots, \mathbf{p}_{i+1}$ is regular and its edge length is $\sqrt{2 q}$. Therefore there is a corresponding $P_{i}$ which contains a translate of $S$ and thus

$$
v_{i}\left(P_{i}\right) \geq v_{i}(S)=c_{i} \cdot n^{i / 2},
$$

where $c_{i}$ is a suitable positive constant depending only on $i$. Comparing (2.1) with (2.2) one can conclude that, if $i$ is fixed and $n$ is sufficiently large, the asymptotic order in (2.1) is optimal.

Now let us introduce two better upper bounds for the areas of the projections.

Theorem 2.2 (Chakerian and Filliman [20]). If $1 \leq i \leq n-1$, for every $i$ dimensional projection $P_{i}$ we have

$$
v_{i}\left(P_{i}\right) \leq \frac{\omega_{i-1}^{i}}{\omega_{i}^{i-1}}\left(\frac{n}{i}\right)^{i / 2} .
$$

Theorem 2.3 (Chakerian and Filliman [20]). If $1 \leq i \leq n-1$, for every $i$ dimensional projection $P_{i}$ we have

$$
v_{i}\left(P_{i}\right) \leq \sqrt{\frac{n !}{(n-i) ! \cdot i !}} .
$$

A polytope is called a zonotope if it is a Minkowski sum of a finite number of segments. Clearly both $I^{n}$ and $P_{i}$ are zonotopes. Let $W_{i}(K)$ denote the $i$-th quermassintegral of an $n$-dimensional convex body $K$. It is well known in Convex Geometry that

$$
\begin{gathered}
W_{n-1}\left(K_{1}+K_{2}\right)=W_{n-1}\left(K_{1}\right)+W_{n-1}\left(K_{2}\right), \\
v_{n}(K) \leq \frac{W_{n-1}(K)^{n}}{\omega_{n}^{n-1}}
\end{gathered}
$$

and, if $K$ is a segment of length $\ell$,

$$
W_{n-1}(K)=\frac{\ell \cdot \omega_{n-1}}{n} .
$$

Usually (2.3) is known as Urysohn's inequality. Then considering $P_{i}$ in $i$-dimensional space, one can deduce

$$
W_{i-1}\left(P_{i}\right) \leq \omega_{i-1} \sqrt{n / i}
$$

and therefore Theorem 2.2.

If $P_{i}$ is an $i$-dimensional zonotope which can be written as a Minkowski sum of segments,

$$
P_{i}=\sum_{j=1}^{n} L_{j},
$$


then it was proved by Shephard [96] that

$$
v_{i}\left(P_{i}\right)=\sum v_{i}\left(\sum_{k=1}^{i} L_{j_{k}}\right),
$$

where the summation is over $\left(\begin{array}{l}n \\ i\end{array}\right)$ sets of indices. Assume that

$$
H^{i}=\left\{\mathbf{x}: x_{j}=0 \text { if } j>i\right\}
$$

and $I$ is an $n$-dimensional unit cube expressed as

$$
I=\sum_{j=1}^{n} \mathbf{u}_{j}
$$

where $\mathbf{u}_{j}=\left(u_{j 1}, u_{j 2}, \cdots, u_{j n}\right)$ are pairwise orthogonal unit segments. Then $U=$ $\left(u_{j k}\right)$ is an $n \times n$ unimodular matrix and, by $(2.4)$,

$$
v_{i}\left(P_{i}\right)=\sum_{\left\{j_{1}, j_{2}, \cdots, j_{i}\right\}}\left\|\begin{array}{cccc}
u_{j_{1} 1} & u_{j_{1} 2} & \cdots & u_{j_{1} i} \\
u_{j_{2} 1} & u_{j_{2} 2} & \cdots & u_{j_{2} i} \\
\vdots & \vdots & \ddots & \vdots \\
u_{j_{i} 1} & u_{j_{i} 2} & \cdots & u_{j_{i} i}
\end{array}\right\| .
$$

Thus, by Cauchy's inequality one can deduce Theorem 2.3 .

Let $D$ be an $i \times i$ sub-matrix of $U$ and let $D^{*}$ denote its algebraic complement. It is known as the Jacobi identity that, if $U^{\prime} U=I_{n}$,

$$
\left|\operatorname{det}\left(D^{*}\right)\right|=|\operatorname{det}(D)| \text {. }
$$

Therefore by (2.5) we can get the following result.

Theorem 2.4 (McMullen [76], Chakerian and Filliman [20]). Suppose that $E^{n}=$ $E^{i} \oplus E^{n-i}$. Let $P$ denote the projection of $I^{n}$ into $E^{i}$ and let $P^{\prime}$ denote the projection of $I^{n}$ into $E^{n-i}$. Then

$$
v_{i}(P)=v_{n-i}\left(P^{\prime}\right) .
$$

Let $\beta(n, i)$ denote the maximum area of the $i$-dimensional projection of $I^{n}$. By Theorem 2.4, the isoperimetric inequality for polygons (see L. Fejes Tóth [33]), and a skillful construction based on complex numbers one can get

$$
\beta(n, 1)=\beta(n, n-1)=\sqrt{n}
$$

and

$$
\beta(n, 2)=\beta(n, n-2)=\cot \left(\frac{\pi}{2 n}\right) .
$$

Based on these results, we list the known values of $\beta(n, i)$ up to $n=7$ in Table 2 .

Similar to the cross sections, Dvoretzky [29] and Larman and Mani [68] proved that, for any fixed $k$, when $n$ is sufficiently large there is a $k$-dimensional projection of $I^{n}$, which is almost spherical. However, no good bound for the number of the $j$-dimensional faces of a $k$-dimensional projection of $I^{n}$ is known. Let $E^{\prime}(n, k, j)$ denote the expected number of the $j$-dimensional faces of a random $k$-dimensional projection of $I^{n}$. Based on a general formula of Affentranger and Schneider [1], it was proved by Böröczky and Henk [16] that

$$
E^{\prime}(n, k, j)=2\left(\begin{array}{c}
n \\
j
\end{array}\right) \sum_{i \geq 0}\left(\begin{array}{c}
n-j \\
k-1-2 i-j
\end{array}\right) \sim 2 \frac{n^{k-1}}{(k-1-j) ! j !} .
$$


TABLE 2 .

\begin{tabular}{|c|c|c|c|c|c|c|c|}
\hline$i$ & 1 & 2 & 3 & 4 & 5 & 6 & 7 \\
\hline$\beta(3, i)$ & $\sqrt{3}$ & $\sqrt{3}$ & 1 & & & & \\
\hline$\beta(4, i)$ & 2 & $\cot (\pi / 8)$ & 2 & 1 & & & \\
\hline$\beta(5, i)$ & $\sqrt{5}$ & $\cot (\pi / 10)$ & $\cot (\pi / 10)$ & $\sqrt{5}$ & 1 & & \\
\hline$\beta(6, i)$ & $\sqrt{6}$ & $\cot (\pi / 12)$ & $? ?$ & $\cot (\pi / 12)$ & $\sqrt{6}$ & 1 & \\
\hline$\beta(7, i)$ & $\sqrt{7}$ & $\cot (\pi / 14)$ & $? ?$ & $? ?$ & $\cot (\pi / 14)$ & $\sqrt{7}$ & 1 \\
\hline
\end{tabular}

\section{INSCRIBED SIMPLICES}

Simplices are another family of important geometric objects. In this section we deal with the following problem.

Problem 3.1. What is the maximum volume $\gamma(n, i)$ of an $i$-dimensional simplex inscribed in an $n$-dimensional unit cube?

Let us start with a simple observation. If $T$ is a tetrahedron with vertices $\mathbf{v}_{1}, \mathbf{v}_{2}$, $\mathbf{v}_{3}$ and $\mathbf{v}_{4}$ and if $\left[\mathbf{v}_{4}^{\prime}, \mathbf{v}_{4}^{*}\right]$ is a segment containing $\mathbf{v}_{4}$ as a relative interior point, then one of the two tetrahedra $T^{\prime}=\operatorname{conv}\left\{\mathbf{v}_{1}, \mathbf{v}_{2}, \mathbf{v}_{3}, \mathbf{v}_{4}^{\prime}\right\}$ and $T^{*}=\operatorname{conv}\left\{\mathbf{v}_{1}, \mathbf{v}_{2}, \mathbf{v}_{3}, \mathbf{v}_{4}^{*}\right\}$ is not smaller than $T$ in volume. As usual $\operatorname{conv}\{X\}$ denotes the convex hull of $X$. Based on this simple observation we can deduce the following fact: For any fixed $i$ and $n, i \leq n$, one of the maximal $i$-dimensional simplices inscribed in $I^{n}$ is a vertex simplex; that is, all its vertices are vertices of $I^{n}$ as well.

Let $S_{i}$ be an $i$-dimensional simplex with vertices $\mathbf{v}_{0}=(0,0, \cdots, 0), \mathbf{v}_{1}=\left(v_{11}\right.$, $\left.v_{12}, \cdots, v_{1 n}\right), \cdots, \mathbf{v}_{i}=\left(v_{i 1}, v_{i 2}, \cdots, v_{i n}\right)$ in the $n$-dimensional Euclidean space $E^{n}$, let $V_{i}=\left(v_{j k}\right)$ denote the corresponding $i \times n$ matrix, let $H$ denote the $i$-dimensional subspace spanned by $\mathbf{v}_{1}, \mathbf{v}_{2}, \cdots, \mathbf{v}_{i}$, and let $H^{\prime}$ denote the $(n-i)$-dimensional subspace which is orthogonal to $H$ in $E^{n}$. Let

$$
I^{n-i}=\sum_{j=i+1}^{n} \mathbf{v}_{j}
$$

be an $(n-i)$-dimensional unit cube, where $\mathbf{v}_{j}$ are pairwise orthogonal unit vectors in $H^{\prime}$, let $V$ be the $n \times n$ matrix $\left(v_{j k}\right)$ and define

$$
S=S_{i} \oplus I^{n-i} .
$$

Then one can deduce that

$$
v_{i}\left(S_{i}\right)=v_{n}(S)=\frac{1}{i !} \sqrt{\operatorname{det}\left(V V^{\prime}\right)}=\frac{1}{i !} \sqrt{\operatorname{det}\left(V_{i} V_{i}^{\prime}\right)} .
$$

Especially, if $S_{i}$ is an $i$-dimensional vertex simplex of $\overline{I^{n}}$, then the corresponding $V_{i}$ is an $i \times n$ binary matrices and therefore

$$
\gamma(n, i)=\frac{1}{i !} \max \sqrt{\operatorname{det}\left(V_{i} V_{i}^{\prime}\right)},
$$

where the maximum is over all $i \times n$ binary matrices $V_{i}$.

By studying a binary $i \times n$ matrix, M. Hudelson, V. Klee and D.G. Larman proved the following general upper bound. 
Theorem 3.1 (Hudelson, Klee and Larman [51]). For $1 \leq i \leq n$ we have

$$
\gamma(n, i) \leq \begin{cases}\frac{1}{i ! 2^{i}} \sqrt{\frac{(i+1)^{i+1} n^{i}}{i^{i}}} & \text { if } i \text { is odd, } \\ \frac{1}{i ! 2^{i}} \sqrt{\frac{(i+2)^{i} n^{i}}{(i+1)^{i-1}}} & \text { if } i \text { is even. }\end{cases}
$$

This theorem can be proved by studying the determinant of $((i+1) I-J) A A^{\prime}$, where $I$ is the $i \times i$ unit matrix, $J$ is the $i \times i$ matrix with all entries being one and $A$ is an $i \times n$ binary matrix. Let $\lambda_{1}, \lambda_{2}, \cdots, \lambda_{i}$ denote the eigenvalues of $((i+$ 1) $I-J) A A^{\prime}$. It is known in Linear Algebra that they are the nonzero eigenvalues of $A^{\prime}((i+1) I-J) A$ as well. Therefore we have

$$
\begin{aligned}
\operatorname{det}\left(((i+1) I-J) A A^{\prime}\right) & \leq \prod_{j=1}^{i} \lambda_{j} \leq\left(\frac{1}{i} \sum_{j=1}^{i} \lambda_{j}\right)^{i} \\
& =\left(\frac{\operatorname{tr}\left(A^{\prime}((i+1) I-J) A\right)}{i}\right)^{i},
\end{aligned}
$$

where $\operatorname{tr}(B)$ is the trace of $B$. By representing the diagonal elements of $A^{\prime}((i+$ 1) $I-J) A$ in terms of the number of ones in the corresponding column of $A$, one can prove the theorem via some basic inequalities.

It was observed by L. Fejes Tóth [33] that the maximal $i$-dimensional simplices contained in the $n$-dimensional unit ball are regular. In fact, the first upper bound in Theorem 3.1 can be deduced from this observation. On the other hand, in both cases of Theorem 3.1, one can construct a corresponding arithmetic series $n=k c_{i}$, where $c_{i}$ is a constant determined by $i$ and $k$ takes all positive integers, such that the upper bounds for $\gamma(n, i)$ are optimal. Therefore we have the following counterpart for Theorem 3.1.

Theorem 3.2 (Neubauer, Watkins and Zeitlin [81]). For any fixed $i$ we have

$$
\lim _{n \rightarrow \infty} \frac{\gamma(n, i)}{n^{i / 2}}= \begin{cases}\frac{1}{i ! 2^{i}} \sqrt{\frac{(i+1)^{i+1}}{i^{i}}} & \text { if } i \text { is odd, } \\ \frac{1}{i ! 2^{i}} \sqrt{\frac{(i+2)^{i}}{(i+1)^{i-1}}} \quad \text { if } i \text { is even. }\end{cases}
$$

When $i=2$ or 3 , we do know the exact values of $\gamma(n, i)$. If $A$ is a $2 \times n$ binary matrix with $k_{1}$ columns identical with $(1,0)^{\prime}, k_{2}$ columns identical with $(0,1)^{\prime}$ and $k_{3}$ columns identical with $(1,1)^{\prime}$, then we have

$$
A A^{\prime}=\left(\begin{array}{cc}
k_{1}+k_{3} & k_{3} \\
k_{3} & k_{2}+k_{3}
\end{array}\right) .
$$

By routine analysis on $\operatorname{det}\left(A A^{\prime}\right)$ one can determine the exact values of $\gamma(n, 2)$ as follows.

Theorem 3.3 (Hudelson, Klee and Larman 51]; Neubauer, Watkins and Zeitlin [81]). If $k=[n / 3]$ and $j=n-3 k$, then

$$
\gamma(n, 2)= \begin{cases}\frac{1}{2} \sqrt{3 k^{2}} & \text { if } j=0, \\ \frac{1}{2} \sqrt{3 k^{2}+2 k} & \text { if } j=1, \\ \frac{1}{2} \sqrt{3 k^{2}+4 k+1} & \text { if } j=2 .\end{cases}
$$


When $i=3$ the proof argument is similar but more complicated. Assume that $T$ is a maximal vertex tetrahedron of $\overline{I^{n}}$ containing $\mathbf{o}$ as one of its vertices. First of all, if three of the four vertices of a vertex tetrahedron of $\overline{I^{n}}$ belong to one facet of $\overline{I^{n}}$, then its volume is smaller than the upper bound listed in the next theorem. Therefore, if $A$ is the corresponding binary $3 \times n$ matrix of one of the maximal vertex tetrahedra, since $\mathbf{o}=(0,0, \cdots, 0)$ is a vertex of $T, A$ has no column identical with $(1,0,0)^{\prime},(0,1,0)^{\prime},(0,0,1)^{\prime}$ or $(1,1,1)^{\prime}$. Then we can prove the following result.

Theorem 3.4 (Hudelson, Klee and Larman [51]; Neubauer, Watkins and Zeitlin [81]). If $k=[n / 3]$ and $j=n-3 k$, then

$$
\gamma(n, 3)=\frac{1}{3} \sqrt{k^{3-j}(k+1)^{j}} .
$$

Remark 3.1. Besides $\gamma(n, 2)$ and $\gamma(n, 3)$, for different $n$ and $i$, no exact value of $\gamma(n, i)$ is known except

$$
\gamma(10,4)=\frac{\sqrt{405}}{4 !}
$$

which was discovered by Hudelson, Klee and Larman [51].

Now we turn to the most interesting and the most important case, $i=n$. For convenience, we define

$$
\kappa_{n}=\max \{\operatorname{det}(B)\},
$$

where the maximum is over all $n \times n$ binary matrices, and

$$
\kappa_{n}^{*}=\max \{\operatorname{det}(A)\},
$$

where the maximum is over all $n \times n$ matrices with \pm 1 entries. By simple transformations it is easy to see that

$$
\kappa_{n+1}^{*}=2^{n} \kappa_{n} .
$$

Therefore, by (3.1), to estimate or determine the value of $\gamma(n, n)$ is equivalent with the corresponding problems for $\kappa_{n}$ and $\kappa_{n+1}^{*}$.

Theorem 3.5 (Hadamard [42], Barba [11, Ehlich 31], 32] and Wojtas [11]). For any $n \times n$ matrix $A$ with \pm 1 entries we have

$$
\operatorname{det}\left(A A^{\prime}\right) \leq \begin{cases}n^{n} & \text { if } n \equiv 0(\bmod 4), \\ (2 n-1)(n-1)^{n-1} & \text { if } n \equiv 1(\bmod 4), \\ 4(n-1)^{2}(n-2)^{n-2} & \text { if } n \equiv 2(\bmod 4), \\ \frac{4 \cdot 11^{6}}{7^{7}} n^{7}(n-3)^{n-7} & \text { if } n \equiv 3(\bmod 4) \text { and } n \geq 63 .\end{cases}
$$

The proof of this theorem is very complicated, especially the fourth case. It is based on detailed analysis of the structure of $A A^{\prime}$ and induction. For example, one can observe that, in the fourth case, every element of $A A^{\prime}$ is $3(\bmod 4)$. Then we can try to get an upper bound for $\operatorname{det}(C)$ instead, where $C$ is a symmetric metric with elements congruent to $3(\bmod 4)$.

The first case is the well-known Hadamard inequality. An $n \times n$ matrix with \pm 1 entries is called a Hadamard matrix if $A A^{\prime}=n I_{n}$. By (3.2) one can easily deduce that Hadamard matrices do exist only if $n \equiv 0(\bmod 4)$. It was conjectured by Paley 84] that the condition is also sufficient. However, this has not been proved yet. On the other hand, it was observed by Grigorév [37] that there is an n-dimensional regular vertex simplex in $I^{n}$ if and only if there exists an $(n+1) \times(n+1)$ Hadamard matrix. It is very surprising indeed that all of the first three upper bounds can be attained at infinitely many $n$, though they are very different.

This theorem can be restated in terms of inscribed simplices in $I^{n}$ as follows. 
Theorem 3.5*. Let $S$ denote an $n$-dimensional simplex contained in $I^{n}$. Then

$$
v_{n}(S) \leq \begin{cases}\frac{1}{n ! 2^{n}} \sqrt{(2 n+1) n^{n}} & \text { if } n \equiv 0(\bmod 4), \\ \frac{1}{(n-1) ! 2^{n-1}} \sqrt{(n-1)^{n-1}} & \text { if } n \equiv 1(\bmod 4), \\ \frac{11^{3}}{n ! 2^{n-1}} \sqrt{\frac{(n-2)^{n-6}(n+1)^{7}}{7^{7}}} & \text { if } n \equiv 2(\bmod 4) \text { and } n \geq 62, \\ \frac{1}{n ! 2^{n}} \sqrt{(n+1)^{n+1}} & \text { if } n \equiv 3(\bmod 4) .\end{cases}
$$

Based on Theorem 3.5 it makes sense to investigate the following problem.

Problem 3.2. Determine the value of

$$
\gamma=\liminf _{n \rightarrow \infty} \frac{\kappa_{n}^{*}}{n^{\frac{n}{2}}} .
$$

Is the sequence $\left\{\kappa_{n}^{*} / n^{\frac{n}{2}}: n=1,2, \cdots\right\}$ dense in $[\gamma, 1]$ ?

Now let us end this section by listing in Table 3 the known values of $\kappa_{n}, \kappa_{n}^{*}$ and $\gamma_{n}=\gamma(n, n)$ up to $n=11$.

TABLE 3 .

\begin{tabular}{|c|c|c|c|c|}
\hline$n$ & 2 & 3 & 4 & 5 \\
\hline$\kappa_{n+1}^{*}$ & 4 & 16 & 48 & 160 \\
\hline$\kappa_{n}=\kappa_{n+1}^{*} / 2^{n}$ & 1 & 2 & 3 & 5 \\
\hline$\gamma_{n}=\kappa_{n} / n !$ & 0.5 & 0.3333333 & 0.125 & 0.0416666 \\
\hline Author & Williamson [110] & Hall, Jr. [48] & Ehlich [31] & Ehlich [31] \\
\hline
\end{tabular}

\begin{tabular}{c|c|c|c|c|c|}
\hline 6 & 7 & 8 & 9 & 10 & 11 \\
\hline 576 & 4096 & $? ?$ & 73728 & $? ?$ & 2985984 \\
\hline 9 & 32 & $? ?$ & 144 & $? ?$ & 1458 \\
\hline 0.0125 & 0.0063492 & $? ?$ & 0.0003968 & $? ?$ & 0.0000365 \\
\hline Williamson [110] & Hall, Jr. [48] & & Ehlich [31] & & Hall, Jr. [48] \\
\hline
\end{tabular}

\section{Triangulations}

Taking a box in hand, one can observe that it has four vertices such that any edge of the box contains at most one of them. By the four planes determined by the triples of these vertices the cube can be divided into five tetrahedra. Then we may ask the following question.

Can one divide the box into four or even fewer tetrahedra?

By a routine argument based on the induced face division and volume estimation one can prove that the answer to this question is "no". 
For convenience, let $V(P)$ denote the set of the vertices of a polytope $P$. A set $\Im=\left\{S_{1}, S_{2}, \cdots, S_{k}\right\}$ of simplices is called a decomposition of $P$ if it satisfies the following conditions.

1. $P=\bigcup_{S_{i} \in \Im} S_{i}$.

2. $\operatorname{int}\left(S_{i}\right) \cap \operatorname{int}\left(S_{j}\right)=\emptyset$ holds for all distinct indices $i$ and $j$.

It will be called a triangulation for $P$ if it satisfies two more conditions.

3. $S_{i} \cap S_{j}$ is a common face of $S_{i}$ and $S_{j}$ whenever it is nonempty.

4. $V\left(S_{i}\right) \subset V(P)$ holds for all indices $i$.

Then we define

$$
\varphi(P)=\min _{\Im}\{\operatorname{card}\{\Im\}\},
$$

where the minimum is over all decompositions of $P$, and

$$
\tau(P)=\min _{\Im}\{\operatorname{card}\{\Im\}\},
$$

where the minimum is over all triangulations for $P$. Especially, we abbreviate $\varphi\left(I^{n}\right)$ and $\tau\left(I^{n}\right)$ to $\varphi_{n}$ and $\tau_{n}$, respectively.

Clearly, triangulations are special cases of decompositions, and therefore

$$
\varphi(P) \leq \tau(P)
$$

holds for all polytopes $P$. Decompositions and triangulations are important in Geometry, Topology and Combinatorics. However, in this section we only focus on the particular case, the cube triangulations. We will deal with two kinds of problems: to find efficient triangulations and to determine the values of $\tau_{n}$. Let us start with introducing several known triangulations for $I^{n}$.

Triangulation I. When $n=2$, we can triangulate $I^{2}$ into two triangles. Assume that $I^{n-1}$ can be triangulated into $(n-1)$ ! simplices. Let $\mathbf{v}$ be a vertex of $I^{n}$ and let $F_{1}, F_{2}, \cdots, F_{n}$ be the $n$ facets which do not contain $\mathbf{v}$. If $\left\{S_{i, j}: j=\right.$ $1,2, \cdots,(n-1) !\}$ are triangulations for $F_{i}$, then the set $\left\{\operatorname{conv}\left\{\mathbf{v} \cup S_{i, j}\right\}: i=\right.$ $1,2, \cdots, n ; j=1,2, \cdots,(n-1) !\}$ will be a triangulation of cardinality $n$ ! for $I^{n}$.

Remark 4.1. In fact, this is the worst triangulation in the sense that it has the maximal cardinality of the simplices. By Theorem $3.5^{*}$ we have

$$
v_{n}(S) \geq \frac{1}{n !}
$$

for all simplices of a triangulation. Therefore

$$
\operatorname{card}\{\Im\} \leq n !
$$

holds for any triangulation $\Im$ of $I^{n}$.

Triangulation II. First of all, we divide $I^{n}$ into several polytopes $P_{1}, P_{2}, \cdots, P_{l}$ such that $V\left(P_{i}\right) \subset V\left(I^{n}\right)$ holds for all indices $i$ and $P_{i} \cap P_{j}$ is a common face of $P_{i}$ and $P_{j}$ whenever it is nonempty. Let $V=\left\{\mathbf{v}_{1}, \mathbf{v}_{2}, \cdots, \mathbf{v}_{2^{n}}\right\}$ be an ordering of the $2^{n}$ vertices of $I^{n}$. For a face $F$ of a polytope we define

$$
i(F)=\min \left\{i: \mathbf{v}_{i} \in F\right\}
$$

and

$$
\mathbf{v}(F)=\mathbf{v}_{i(F)} .
$$

For each sequence of faces $P=F_{n} \supset F_{n-1} \supset \cdots \supset F_{0} \neq \emptyset$ such that $\mathbf{v}\left(F_{i+1}\right) \notin F_{i}$ holds for $0 \leq i \leq n-1$ we define a simplex $\operatorname{conv}\left\{\mathbf{v}\left(F_{n}\right), \mathbf{v}\left(F_{n-1}\right), \cdots, \mathbf{v}\left(F_{0}\right)\right\}$. Then 
all simplices of this kind produce a triangulation $\Im$ for $I^{n}$. In this way, by dividing $I^{n}$ into suitable polytopes and choosing a suitable ordering $V$, Sallee [93] was able to improve (4.1) to

$$
\tau_{n}=o(1) \cdot n !
$$

Triangulation III. Let $S^{i}$ denote an $i$-dimensional simplex. It was proved by Billera, Cushman and Sanders 13 that

$$
\tau\left(S^{k} \oplus S^{l}\right)=\frac{(k+l) !}{k ! \cdot l !} .
$$

If $\Im_{k}=\left\{S_{1}^{k}, S_{2}^{k}, \cdots, S_{\tau_{k}}^{k}\right\}$ is a triangulation for $I^{k}$ and $\Im_{l}=\left\{S_{1}^{l}, S_{2}^{l}, \cdots, S_{\tau_{l}}^{l}\right\}$ is a triangulation for $I^{l}$, then

$$
I^{k+l}=\bigcup_{i, j} S_{i}^{k} \oplus S_{j}^{l}
$$

Let $\Im_{i, j}$ be a triangulation for $S_{i}^{k} \oplus S_{j}^{l}$. Then $\bigcup_{i, j} \Im_{i, j}$ will be a triangulation for $I^{k+l}$. Based on this observation, Haiman [45] and Orden and Santos 82] were able to prove the following theorem. So far it is the best known upper bound for $\tau_{n}$.

Theorem 4.1. When $n$ is large,

$$
\tau_{n} \leq 0.816^{n} \cdot n !
$$

On the other hand, it is well known (see Theorem $3.5^{*}$ ) that

$$
v_{n}(S) \leq \frac{(n+1)^{\frac{n+1}{2}}}{2^{n} \cdot n !}
$$

holds for all simplices of a triangulation for $I^{n}$. Therefore we have

$$
\tau_{n} \geq \frac{2^{n} n !}{(n+1)^{\frac{n+1}{2}}} .
$$

In the spherical model of Hyperbolic Geometry, the measure of a set $A$ contained in the unit ball is defined by

$$
\varpi(A)=\int_{A}\left(1-\|\mathbf{x}\|^{2}\right)^{-\frac{n+1}{2}} d \mathbf{x} .
$$

It was conjectured by Thurston 106] and proved by Haagerup and Munkholm [41. that

$$
\varpi(S) \leq\left(\frac{n+1}{n-1}\right)^{\frac{n+1}{2}} \frac{1}{n !}
$$

holds for any simplex $S$ inscribed in the unit ball. By proving

$$
\varpi(I) \geq\left(\frac{4}{n}\right)^{\frac{n}{2}}\left(\frac{3}{2}\right)^{\frac{n+1}{2}}
$$

for the cube $I$ inscribed in the unit ball, Smith was able to improve (4.4) into the following theorem. So far it is the best known lower bound for $\tau_{n}$.

Theorem 4.2 (Smith [98]).

$$
\tau_{n} \geq \frac{6^{\frac{n}{2}} \cdot n !}{2 \cdot(n+1)^{\frac{n+1}{2}}} .
$$


Comparing Theorem 4.2 with Theorem 4.1, one notices that the gap between the known upper bound and the known lower bound for $\tau_{n}$ is still huge. As for the exact values of $\tau_{n}$ we have the following results.

Theorem 4.3 (Mara 74, Cottle [23], Sallee [93], Lee 69], Hughes [53, Hughes and Anderson [55]).

\begin{tabular}{|c|c|c|c|c|c|c|}
\hline$n$ & 2 & 3 & 4 & 5 & 6 & 7 \\
\hline$\tau_{n}$ & 2 & 5 & 16 & 67 & 308 & 1493 \\
\hline
\end{tabular}

As one can imagine the cases $n=5,6$ and 7 were achieved by complicated linear and integer programs, with computer aid. The $n=4$ case can be deduced by volume estimation and dealing with several cases. It also can be deduced by $f$-vectors and $h$-vectors.

Remark 4.2. In 2000, Below, Brehm, De Loera and Richter-Gebert [12] discovered that there are three-dimensional polytopes $P$ satisfying

$$
\varphi(P) \neq \tau(P) \text {. }
$$

However, we do not know if

$$
\tau_{n}=\varphi_{n}
$$

holds for all $n$. So far this is known up to $n=5$.

\section{0/1 POLYTOPES}

0/1 polytopes are convex hulls of subsets of the vertex set of $\overline{I^{n}}$. Besides their own geometric and combinatorial interest, $0 / 1$ polytopes do provide intuitive models to Coding Theory, Combinatorial Optimization, etc. There are several fundamental problems concerning the geometry and the combinatorics of $0 / 1$ polytopes. For example,

Problem 5.1. Determine or estimate the number of different classes of all $n$ dimensional 0/1 polytopes (with respect to a certain equivalence).

Problem 5.2. Determine or estimate the maximal number of the $i$-faces of an $n$-dimensional $0 / 1$ polytope.

Problem 5.3. Given $n$ and $s$. What is the maximal number $A(n, s)$ such that there is an $n$-dimensional $0 / 1$ polytope with $A(n, s)$ vertices and the minimal distance between them is not smaller than $\sqrt{s}$ ?

Let $\phi(n)$ denote the number of the $n$-dimensional $0 / 1$ polytopes reduced from $\overline{I^{n}}$. By simple combinatorial arguments one can show that

$$
c \cdot 2^{2^{n}}<\phi(n)<2^{2^{n}}
$$

holds for some suitable constant $c$.

There are several types of classification for $0 / 1$ polytopes based on distinct equivalence relations. For example, the classification based on affine equivalence, congruence, combinatorial equivalence or 0/1 equivalence. The first two are well known in geometry. Now, let us briefly introduce the third and the fourth ones. Let $\mathcal{F}_{P}$ denote the face lattice of a polytope $P$, that is, the set of all faces of $P$ partially ordered by inclusion. Two polytopes $P_{1}$ and $P_{2}$ are combinatorially equivalent if $\mathcal{F}_{P_{1}}$ is isomorphic to $\mathcal{F}_{P_{2}}$. Two $0 / 1$ polytopes $P_{1}$ and $P_{2}$ are $0 / 1$ equivalent if one can be transformed into the other by a symmetry of the unit cube $\overline{I^{n}}$. 
Restricting to the family of $n$-dimensional $0 / 1$ polytopes, we have the following relations between $0 / 1$ equivalence $\left(E_{1}\right)$, congruence $\left(E_{2}\right)$, affine equivalence $\left(E_{3}\right)$ and combinatorial equivalence $\left(E_{4}\right)$.

Theorem 5.1 (Ziegler [115]).

$$
E_{1} \Longrightarrow E_{2} \Longrightarrow E_{3} \Longrightarrow E_{4} \text {. }
$$

This assertion is easy to prove. However, the converse to any of the three implications is false. It is easy to get $0 / 1$ polytopes that are affinely equivalent but not congruent. To show the other cases we have the following examples, both from Ziegler [115.

Example 5.1. Let $S_{1}$ be a five-dimensional simplex with vertices $\mathbf{u}_{1}=(0,0,0,0,0)$, $\mathbf{u}_{2}=(0,0,1,1,0), \mathbf{u}_{3}=(0,1,0,1,0), \mathbf{u}_{4}=(1,0,0,1,0), \mathbf{u}_{5}=(0,1,1,0,0)$ and $\mathbf{u}_{6}=$ $(0,1,1,0,1)$ and let $S_{2}$ be a five-dimensional simplex with vertices $\mathbf{v}_{1}=(0,0,0,0,0)$, $\mathbf{v}_{2}=(0,0,1,1,0), \mathbf{v}_{3}=(0,1,0,1,0), \mathbf{v}_{4}=(0,1,1,0,0), \mathbf{v}_{5}=(1,0,0,1,0)$ and $\mathbf{v}_{6}=(1,0,0,1,1)$. It is easy to verify that

$$
\left\|\mathbf{u}_{i}-\mathbf{u}_{j}\right\|=\left\|\mathbf{v}_{i}-\mathbf{v}_{j}\right\|
$$

holds for all index pairs $\{i, j\}$. Thus $S_{1}$ and $S_{2}$ are congruent. However, $S_{1}$ and $S_{2}$ are not $0 / 1$ equivalent.

Example 5.2. Let $P_{1}$ be a five-dimensional polytope with vertices $\mathbf{u}_{1}=(0,0,0$, $0,0), \mathbf{u}_{2}=(1,0,0,0,0), \mathbf{u}_{3}=(0,1,0,0,0), \mathbf{u}_{4}=(0,0,1,0,0), \mathbf{u}_{5}=(0,0,0,1,0)$, $\mathbf{u}_{6}=(0,0,0,0,1)$ and $\mathbf{u}_{7}=(1,1,1,1,1)$ and let $P_{2}$ be a five-dimensional polytope with vertices $\mathbf{v}_{1}=(0,0,0,0,0), \mathbf{v}_{2}=(1,1,0,0,0), \mathbf{v}_{3}=(0,1,1,0,0), \mathbf{v}_{4}=$ $(0,0,1,1,0), \mathbf{v}_{5}=(0,0,0,1,1), \mathbf{v}_{6}=(1,0,0,0,1)$ and $\mathbf{v}_{7}=(1,1,1,1,1)$. In fact, both $P_{1}$ and $P_{2}$ are bipyramids over a four-dimensional simplex. Therefore they are combinatorially equivalent. However, since in $P_{1}$ and $P_{2}$ the main diagonals are divided by the simplex in the ratios $1: 4$ and $2: 3$ respectively, they are not affinely equivalent.

Let $\phi_{1}(n), \phi_{2}(n), \phi_{3}(n)$ and $\phi_{4}(n)$ denote the numbers of the different classes of $n$-dimensional $0 / 1$ polytopes with respect to $0 / 1$ equivalence, congruence, affine equivalence and combinatorial equivalence, respectively. It follows from Theorem 5.1 that

$$
\phi_{4}(n) \leq \phi_{3}(n) \leq \phi_{2}(n) \leq \phi_{1}(n) \leq \phi(n) .
$$

For large $n$ to determine the exact values of $\phi_{i}(n)$ or even $\phi(n)$ is a very hard job. So far, our knowledge of this kind is very limited. We list the known ones in Table 4. Especially, we point out that the values of $\phi(5)$ and $\phi_{1}(5)$ were discovered by Aichholzer [3].

It follows by (5.1) and (5.2) that

$$
\phi_{i}(n)<2^{2^{n}}
$$

holds for all $i=1,2,3$ and 4 . These upper bounds are certainly not optimal. However, so far no essentially better upper bound for $\phi_{i}(n)$ is known. As a counterpart of (5.3) we have the following lower bound for $\phi_{i}(n)$.

Theorem 5.2 (Ziegler [115]). When $n \geq 6$ we have

$$
\phi_{i}(n) \geq 2^{2^{n-2}}
$$

for all $i=1,2,3$ and 4 . 
TABLE 4.

\begin{tabular}{|c|c|c|c|c|c|}
\hline$n$ & $\phi(n)$ & $\phi_{1}(n)$ & $\phi_{2}(n)$ & $\phi_{3}(n)$ & $\phi_{4}(n)$ \\
\hline 2 & 5 & 2 & 2 & 2 & 2 \\
\hline 3 & 151 & 12 & 12 & 8 & 8 \\
\hline 4 & 60879 & 347 & 347 & $? ?$ & 172 \\
\hline 5 & 4292660729 & 1226525 & $? ?$ & $? ?$ & $? ?$ \\
\hline
\end{tabular}

Let $F_{i}^{0}$ and $F_{i}^{1}$ denote the facets of $\overline{I^{n}}$ given by $x_{i}=0$ and $x_{i}=1$, respectively. For convenience we will call $F_{n}^{0}$ the bottom facet, $F_{n}^{1}$ the top facet and all the others vertical facets of $\overline{I^{n}}$. For $n \geq 3$ let $\mathcal{P}_{n}$ denote the family of $0 / 1$ polytopes $P$ reduced from $\overline{I^{n}}$ and satisfying the following conditions.

1. It contains the whole bottom facet of $\overline{I^{n}}$.

2. It contains both $\mathbf{e}_{n}=(0,0, \cdots, 1)$ and $\mathbf{e}=(1,1, \cdots, 1)$.

3. It contains neither $\mathbf{e}_{n}+\mathbf{e}_{1}=(1,0, \cdots, 1)$ nor $\mathbf{e}-\mathbf{e}_{1}=(0,1, \cdots, 1)$.

Clearly all the polytopes contained in $\mathcal{P}_{n}$ are $n$-dimensional and

$$
\operatorname{card}\left\{\mathcal{P}_{n}\right\}=\sum_{i=0}^{2^{n-1}-4}\left(\begin{array}{c}
2^{n-1}-4 \\
i
\end{array}\right)=2^{2^{n-1}-4} .
$$

On the other hand, assuming that $\mathcal{P}_{n}$ can be divided into combinatorially equivalent classes $\mathcal{C}_{1}, \mathcal{C}_{2}, \cdots, \mathcal{C}_{k}$, by detailed analysis one can prove that

$$
\operatorname{card}\left\{\mathcal{C}_{j}\right\} \leq 2^{n-1} \cdot(n-1) !
$$

holds for all $j=1,2, \cdots, k$. Thus the theorem follows by (5.4) and (5.5).

Next we discuss some known results about Problem 5.2. Let $\varsigma(n, k)$ denote the maximal number of the $k$-dimensional faces of an $n$-dimensional $0 / 1$ polytope, and especially abbreviate $\varsigma(n, n-1)$ to $\varsigma(n)$. The known exact values of $\varsigma(n)$ are listed in Table 5 (see Ziegler [115).

TABLE 5 .

\begin{tabular}{|c|c|c|c|c|}
\hline$n$ & 2 & 3 & 4 & 5 \\
\hline$\varsigma(n)$ & 4 & 8 & 16 & 40 \\
\hline
\end{tabular}

Let $\mathbf{e}_{1}, \mathbf{e}_{2}, \cdots, \mathbf{e}_{n}$ denote the $n$ vectors of an orthonormal basis of $E^{n}$ and write $\mathbf{e}=(1,1, \cdots, 1)$. Then it is easy to see that

$$
T_{n}=\operatorname{conv}\left\{\mathbf{e}_{1}, \mathbf{e}-\mathbf{e}_{1}, \cdots, \mathbf{e}_{n}, \mathbf{e}-\mathbf{e}_{n}\right\}
$$

is centrally symmetric with respect to the center of $\overline{I^{n}}$ and therefore it is an $n$ dimensional $0 / 1$ cross polytope. By this example one can easily deduce that

$$
\varsigma(n) \geq 2^{n} \text {. }
$$

In fact, for sufficiently large $n$, by a similar technique this lower bound can be improved into

$$
\varsigma(n) \geq 3.6^{n} \text {. }
$$


Very recently, by a complicated random method, I. Bárány and A. Pór proved the following result. So far this is the best known lower bound for $\varsigma(n)$.

Theorem 5.3 (Bárány and Pór [10]). When $n$ is sufficiently large, we have

$$
\varsigma(n) \geq\left(\frac{c \cdot n}{\log n}\right)^{0.25 n}
$$

for some suitable positive constant $c$.

As a counterpart for Theorem 5.3, we have the following upper bound for $\varsigma(n)$.

Theorem 5.4 (Fleiner, Kaibel and Rote [34]). There is a positive number c such that

$$
\varsigma(n) \leq c \cdot(n-2) !
$$

Let $P$ denote an $n$-dimensional $0 / 1$ polytope with $\varsigma(n)$ facets. The proof of this theorem is based on two key ideas. First, if $\varsigma(n) \leq(n-2)$ !, there is nothing to prove; if $\varsigma(n) \geq(n-2)$ !, then try to prove $\varsigma(n) \leq c \cdot(n-2)$ !. Second, for any facet $F$ of $P$ there is a normal vector $\mathbf{u}$ of the form

$$
\mathbf{u}=(n-1) ! \cdot\left(u_{1}, u_{2}, \cdots, u_{n}\right),
$$

where the $u_{i}$ are integers. Then consider the sum of the absolute norm of all these vectors.

Remark 5.1. Comparing Theorem 5.3 with Theorem 5.4, it is easy to see that

$$
c_{1} n \cdot \log n \leq \log \varsigma(n) \leq c_{2} n \cdot \log n
$$

holds for two constants $c_{1}$ and $c_{2}$. From this point of view, both bounds are quite good.

Next we introduce some known results pertaining to Problem 5.3. In fact, it is a basic problem in Coding Theory. Let $F_{2}$ denote the binary field and let $H_{2}^{n}$ denote the Hamming space, the $n$-dimensional linear space over $F_{2}$ and associated with the Hamming metric

$$
\|\mathbf{x}, \mathbf{y}\|_{H}=\operatorname{card}\left\{i: x_{i} \neq y_{i}\right\} .
$$

We notice that

$$
\|\mathbf{x}, \mathbf{y}\|=\sqrt{\|\mathbf{x}, \mathbf{y}\|_{H}}
$$

holds whenever both $\mathbf{x}$ and $\mathbf{y}$ belong to $H_{2}^{n}$.

Usually, a point $\mathbf{c} \in H_{2}^{n}$ will be called a binary codeword, a subset $C$ of $H_{2}^{n}$ will be called a binary code and the minimum Hamming distance between distinct points in $C$ is called the separation of $C$, denoted by $s(C)$. In addition, for convenience, a code of length $n$, size $m$ and separation $s$ will be called an $(n, m, s)$-code. Then we can restate Problem 5.3 as follows.

Problem 5.3*. Given $n$ and $s$. What is the maximal number $A(n, s)$ such that there is a code $C$ in $H_{2}^{n}$ with cardinality $A(n, s)$ and separation $s ?$

Roughly speaking, an information transmission process can be described as follows. First, design a code $C$ and encode the information into codewords. Second, transmit the codewords through a channel to a receiver. Since the channel may add errors, the received words (in $H_{2}^{n}$ ) perhaps are not the sent ones. Third, design a decoder to eliminate the errors. In this step, if a received word $\mathbf{w}$ is not a codeword 
of $C$, then it will be replaced by one of its closest codewords c. It is easy to imagine that if $s=s(C)$ is relatively large, then the errors caused by the transmitting channel will be eliminated more easily. On the other hand, if card $\{C\}$ is relatively large, then the code is more efficient. Therefore it is easy to see that Problem 5.3* is indeed a key problem in Coding Theory.

Let us start with some basic results about $A(n, s)$. First of all, it is obvious that

$$
A(n, 1)=2^{n}
$$

and

$$
A(n, n)=2 .
$$

Second, if $C$ is a binary $(n, m, s)$-code with $m=A(n, s)$ and if for $i=0$ and 1 we define

$$
C_{i}=\left\{\mathbf{c} \in C: c_{1}=i\right\},
$$

then $C_{0}$ will reduce to an $\left(n-1, m_{0}, s\right)$-code and $C_{1}$ will reduce to an $\left(n-1, m_{1}, s\right)$ code. Since one of them has a cardinality not smaller than $A(n, s) / 2$, we have

$$
A(n, s) \leq 2 A(n-1, s) .
$$

Third, if $C$ is a binary $(n, m, 2 k-1)$-code with $m=A(n, 2 k-1)$, by adding an overall parity check to each codeword one can produce an $(n+1, m, 2 k)$-code. On the other hand, suppose that $C$ is a binary $(n+1, m, 2 k)$-code with $m=$ $A(n+1,2 k)$, by puncturing $C$ in a position at which two codewords disagree one gets an $(n, m, 2 k-1)$-code with $m=A(n+1,2 k)$. Thus we have

$$
A(n, 2 k-1)=A(n+1,2 k) .
$$

Now let us introduce several well-known bounds for $A(n, s)$.

Theorem 5.5 (The Gilbert-Varshamov bound [36] and [109]).

$$
A(n, s) \geq \frac{2^{n}}{\sum_{k=0}^{s-1}\left(\begin{array}{l}
n \\
k
\end{array}\right)} .
$$

Theorem 5.6 (The Hamming bound).

$$
A(n, s) \leq \frac{2^{n}}{\sum_{k=0}^{s^{\prime}-1}\left(\begin{array}{l}
n \\
k
\end{array}\right)},
$$

where $s^{\prime}=[(s-1) / 2]$.

Theorem 5.7 (The Elias bound). Assume that $r$ is an integer satisfying $r \leq n / 2$ and $r^{2}-n r+n s / 2>0$. Then

$$
A(n, s) \leq \frac{n s}{2 r^{2}-2 n r+n s} \cdot \frac{2^{n}}{\sum_{k=0}^{r}\left(\begin{array}{l}
n \\
k
\end{array}\right)} .
$$

Theorem 5.8 (Delsarte [26] and [27]). When $s$ is even (if it is odd, then apply (5.6)) we have

$$
\begin{aligned}
& A(n, s) \leq \max \left\{\sum_{j=0}^{n} a_{j}: a_{0}=1 ; a_{j}=0 \text { for } 1 \leq j \leq s \text { or } j\right. \text { is odd; } \\
& \left.a_{j} \geq 0 ; \sum_{j=0}^{n} a_{j} K_{i}(j) \geq 0 \text { for } 0 \leq i \leq n\right\} \text {, }
\end{aligned}
$$


where $K_{i}(x)$ are Krawtchouk polynomials defined as

$$
K_{i}(x)=\sum_{j=0}^{i}(-1)^{j}\left(\begin{array}{l}
x \\
j
\end{array}\right)\left(\begin{array}{c}
n-x \\
i-j
\end{array}\right) .
$$

Theorem 5.5 and Theorem 5.6 can be easily proved by ideas of sphere packing and sphere covering, respectively. However, the proofs for Theorem 5.7 and Theorem 5.8 are complicated, especially Theorem 5.8. Since they are well known, we refer the interested readers to the standard books in Coding Theory such as Pless and Huffman [87] or van Lint [108]. As one can notice from the above theorems, the gap between the known lower bound and the best known upper bound is still remarkable.

We list some known values of $A(n, s)$ in Table 6 , which is quoted from Sloane 97.

TABLE 6 .

\begin{tabular}{|c|c|c|c|c|c|c|c|c|c|c|c|}
\hline$n$ & 5 & 6 & 7 & 8 & 9 & 10 & 11 & 12 & 13 & 14 & 15 \\
\hline$A(n, 3)$ & 4 & 8 & 16 & 20 & 40 & 72 & 144 & 256 & 512 & 1024 & 2048 \\
\hline$A(n, 5)$ & 2 & 2 & 2 & 4 & 6 & 12 & 24 & 32 & 64 & 128 & 256 \\
\hline$A(n, 7)$ & - & - & 2 & 2 & 2 & 2 & 4 & 4 & 8 & 16 & 32 \\
\hline
\end{tabular}

Remark 5.2. Concerning the volume of a $0 / 1$ polytope, there is an interesting but rather isolated result. Let $\sigma(n, m)$ denote the average volume of the $0 / 1$ polytopes in $E^{n}$ and with $m$ vertices. It was shown by Dyer, Füredi and McDiarmid [30] that, letting $\epsilon$ be any positive number and writing $\alpha=2 / \sqrt{e}$,

$$
\lim _{n \rightarrow \infty} \sigma(n, m)= \begin{cases}1 & \text { if } m \geq(\alpha+\epsilon)^{n} \\ 0 & \text { if } m \leq(\alpha-\epsilon)^{n}\end{cases}
$$

\section{Minkowski's CONJECTURE}

Let $I^{2}+\Lambda$ be a lattice tiling in $E^{2}$ and let $\mathbf{b}_{1}=(1, \beta) \in \Lambda$ be a suitable point such that $I^{2}+\mathbf{b}_{1}$ meets $I^{2}$ at its boundary. If $\beta=0$, then $I^{2}+\mathbf{b}_{1}$ meets $I^{2}$ at a whole edge. If $\beta \neq 0$, since $I^{2}+\Lambda$ is a tiling in $E^{2}$, then we have $\mathbf{b}_{2}=(0,1) \in \Lambda$ and therefore $I^{2}+\mathbf{b}_{2}$ meets $I^{2}$ at a whole edge. As a conclusion, if $I^{2}+\Lambda$ is a lattice tiling of $E^{2}$, then $I^{2}$ meets one of its neighbors at a whole edge. By a similar argument this result can be easily extended to three dimensions. In 1896 Minkowski 78] discovered this fact and promised to prove a similar statement in $E^{n}$. However, the promised proof did not appear. For this reason the $n$-dimensional case is known as Minkowski's conjecture. For convenience, we will call two $n$-dimensional cubes a twin whenever they share a whole facet.

Minkowski's conjecture. Every lattice tiling $I^{n}+\Lambda$ of $E^{n}$ has twins.

To approach this simple sounding conjecture in high dimensions, T. Schmidt proved the following intermediate result, which plays a very important role in the final proof of this conjecture. 
Lemma 6.1 (Schmidt 95]). If there is a lattice tiling $I^{n}+\Lambda$ of $E^{n}$ without a twin, then there is a rational lattice tiling $I^{n}+\Lambda^{\prime}$ without a twin.

Of course, a rational lattice means all the lattice points have rational coordinates, or in other words it has a rational basis. Clearly, if $I^{n}+\left(a_{1}, \cdots, a_{n}\right)$ touches $I^{n}$ at its boundary and if $a_{1}$ is irrational, then $-1<a_{1}<1$ and therefore one can find a small $\epsilon$ such that $I^{n}+\left(a_{1}+\epsilon, \cdots, a_{n}\right)$ touches $I^{n}$ at its boundary and $a_{1}+\epsilon$ is rational. Based on this observation the lemma can be proved by detailed analysis.

Let $\Lambda$ be a rational lattice with a basis $\mathbf{b}_{1}, \cdots, \mathbf{b}_{n}$, where

$$
\mathbf{b}_{i}=\left(\frac{c_{i 1}}{d_{i 1}}, \cdots, \frac{c_{i n}}{d_{i n}}\right)
$$

and where $c_{i j}$ and $d_{i j}$ are integers. Let $q_{j}$ denote the common multiple of $d_{1 j}, \cdots$, $d_{n j}$, and let $\bar{\Lambda}$ denote the lattice generated by $\overline{\mathbf{b}_{1}}=\frac{1}{q_{1}} \mathbf{e}_{1}, \cdots, \overline{\mathbf{b}_{n}}=\frac{1}{q_{n}} \mathbf{e}_{n}$, where $\mathbf{e}_{i}$ indicates the $i$-th unit axis. Then $\bar{\Lambda} / \Lambda$ can be uniquely expressed as

$$
\sum_{i=1}^{n} z_{i} \overline{\mathbf{b}_{i}}
$$

with $z_{i} \in Z$ and $0 \leq z_{i} \leq q_{i}-1$. Especially, we have $\mathbf{e}_{i} \in \Lambda$ for some $i$ whenever $I^{n}+\Lambda$ has a twin. Therefore Hajós [46 was able to reformulate Minkowski's conjecture into the following version.

Minkowski's conjecture in algebraic version. Let $G$ be a finite abelian group with unit $\mathbf{1}$. If $\mathbf{g}_{1}, \cdots, \mathbf{g}_{n}$ are elements of $G$ and $q_{1}, \cdots, q_{n}$ are positive integers such that each element of $G$ can be uniquely written in the form

$$
\prod_{i=1}^{n} \mathbf{g}_{i}^{z_{i}}, \quad 0 \leq z_{i} \leq q_{i}-1
$$

then $\mathbf{g}_{i}^{q_{i}}=\mathbf{1}$ for some $i$ with $1 \leq i \leq n$.

Let $\Re(G)$ denote the group ring generated by $G$. In other words,

$$
\Re(G)=\left\{\sum z_{i} \mathbf{g}_{i}: z_{i} \in Z ; \mathbf{g}_{i} \in G\right\}
$$

in which the addition is defined by

$$
\sum z_{i} \mathbf{g}_{i}+\sum z_{i}^{\prime} \mathbf{g}_{i}=\sum\left(z_{i}+z_{i}^{\prime}\right) \mathbf{g}_{i}
$$

and the multiplication is defined by

$$
\left(\sum z_{i} \mathbf{g}_{i}\right)\left(\sum z_{i}^{\prime} \mathbf{g}_{i}\right)=\sum\left(\sum_{\mathbf{g}_{j} \mathbf{g}_{k}=\mathbf{g}_{i}} z_{j} z_{k}^{\prime}\right) \mathbf{g}_{i} .
$$

In 1942, by deep study in group rings, Hajós [46] was able to prove Minkowski's conjecture.

Theorem 6.1 (The Minkowski-Hajós theorem). Every lattice tiling $I^{n}+\Lambda$ of $E^{n}$ has twins.

Before Hajós' proof, Jansen [57], Schmidt [95], Keller [61], [62] and Perron [86] made different approaches to Minkowski's conjecture and proved it for $n \leq 9$. Perron's proof was based on the observation that, if the center of a cube of a lattice tiling is not the origin, at least one of its coordinates is a nonzero integer. 
It is known that every tile (translative) is a polytope and the unit cube is the most regular one. Based on Theorem 6.1 it is reasonable to make the following conjecture.

Conjecture 6.1. Let $T$ be a tile. Every lattice tiling $T+\Lambda$ of $E^{n}$ has two translates sharing a facet.

Besides the geometric version and the algebraic version, the Minkowski-Hajós theorem can also be stated as a version of Diophantine equations (see Kolountzakis 63) and as a version of Diophantine approximation. In fact, Minkowski did first state his conjecture in the form of Diophantine approximation. Ten years later he restated it in the language of geometry.

Theorem 6.1*. If $A$ is an $n \times n$ matrix such that $\operatorname{det} A=1$, then there is a $\mathbf{z} \in Z^{n} \backslash\{\mathbf{o}\}$ such that

$$
\|A \mathbf{z}\|_{\infty}<1
$$

unless $A$ has an integral row. Here, as usual,

$$
\|\mathbf{x}\|_{\infty}=\max \left\{\left|x_{i}\right|: 1 \leq i \leq n\right\} .
$$

By induction one can even restate Theorem 6.1 in the following version.

Theorem 6.1**. If $I^{n}+\Lambda$ is a lattice tiling of $E^{n}$ and $\Lambda=A Z^{n}$, then there is a unimodular integral matrix $U$ such that

$$
A U=\left(\begin{array}{ccccc}
1 & 0 & 0 & \cdots & 0 \\
\alpha_{21} & 1 & 0 & \cdots & 0 \\
\alpha_{31} & \alpha_{32} & 1 & \cdots & 0 \\
\vdots & \vdots & \vdots & \ddots & \vdots \\
\alpha_{n 1} & \alpha_{n 2} & \alpha_{n 3} & \cdots & 1
\end{array}\right),
$$

where $\left|\alpha_{i j}\right|<1$ holds for all $i$ and $j$.

The unit cube $I^{n}=I^{1} \oplus I^{1} \oplus \cdots \oplus I^{1}$ is a very special cylinder. Thus, based on Theorem $6.1^{* *}$ one can ask the following question which will be useful in the study of the packing and covering of a general convex body.

Problem 6.1. Let $T_{i}$ denote a tile in $E^{n_{i}}$. If $T_{1} \oplus T_{2}+\Lambda$ is a lattice tiling of $E^{n_{1}+n_{2}}$ and $\Lambda=A Z^{n_{1}+n_{2}}$, is there always a unimodular integral matrix $U$ such that

$$
A U=\left(\begin{array}{ll}
A_{11} & A_{12} \\
A_{21} & A_{22}
\end{array}\right),
$$

where $A_{12}$ is an $n_{1} \times n_{2}$ zero matrix?

\section{KelleR's CONJECTURE AND FurtwänGLER's CONJECTURE}

In 1930, besides proving Minkowski's conjecture for some special dimensions, Keller 61] made an even more ambitious conjecture.

Keller's conjecture. Every translative tiling $I^{n}+X$ of $E^{n}$ has a twin.

In fact, by elementary methods like that at the beginning of Section 6, one can easily deduce this conjecture in $E^{2}$ and $E^{3}$. In this direction, by complicated arguments O. Perron even proved Keller's conjecture for $n \leq 6$ (Keller [62] contained a proof sketch for this result). 
Theorem 7.1 (Keller [62], Perron [85]). When $n \leq 6$, every translative tiling $I^{n}+X$ of $E^{n}$ has a twin.

Similar to Minkowski's conjecture, Keller's conjecture also has an algebraic version, which was discovered by Hajós [47] in 1950.

Keller's conjecture in algebraic version. Let $G$ be an abelian group with basis elements $\mathbf{g}_{1}, \cdots, \mathbf{g}_{n}$ of orders $2 q_{1}, \cdots, 2 q_{n}$ respectively. If $G=H A_{1} \cdots A_{n}$ is a factorization, where $|H|=2^{n}$ and $A_{i}=\left\{\mathbf{1}, \mathbf{g}_{i}, \cdots, \mathbf{g}_{i}^{q_{i}-1}\right\}$, then

$$
H^{-1} H \cap\left\{\mathbf{g}_{1}^{q_{1}}, \cdots, \mathbf{g}_{n}^{q_{n}}\right\} \neq \emptyset .
$$

In 1940 O. Perron proposed an idea to reduce Keller's conjecture into finitely many cases for each $n$. In 1986 S. Szabó made this idea more explicit and proved the following statement.

Lemma 7.1 (Perron 85] and Szabó [104]). If Keller's conjecture is false in $E^{n}$, then there exists a counterexample tiling $I^{m}+X$ in some $E^{m}$ with $m \geq n$ such that $X \subset \frac{1}{2} Z^{m}$ and $X$ is periodic with a period lattice containing $2 Z^{m}$.

Later, K. Corrádi and S. Szabó introduced a graph $G_{n}$ and a graph-theoretic version of this lemma. The vertices of $G_{n}$ are vectors of length $n$ with entries from $\{0,1,2,3\}$. Two such vectors $\mathbf{u}$ and $\mathbf{v}$ are adjacent if and only if $u_{i}-v_{i} \equiv 2(\bmod$ 4) for some $i$ and $u_{j} \neq v_{j}$ for some $j, j \neq i$.

Lemma 7.1* (Corrádi and Szabó 22]). There is a counterexample for Keller's conjecture if and only if for some $n$ the graph $G_{n}$ has a clique of size $2^{n}$.

These are very important steps towards the solution for this long-standing conjecture. In 1992, by constructing such graphs, J.C. Lagarias and P.W. Shor disproved Keller's conjecture for $n \geq 10$. In 2002 J. Mackey improved it to $n \geq 8$. Thus we have the following theorem.

Theorem 7.2 (Lagarias and Shor [66], Mackey [73]). Whenever $n \geq 8$, there is a translative tiling $I^{n}+X$ of $E^{n}$ which has no twin.

Comparing Theorem 7.2 with Theorem 7.1 one notices that $n=7$ is the only open case for Keller's conjecture now.

In 1994 J.C. Lagarias and P.W. Shor improved this result by considering the maximal dimension of the common face of two touching cubes in a translative tiling. Let $\xi_{n}$ denote the largest integer such that every translative tiling $I^{n}+X$ of $E^{n}$ contains two cubes which have a common face of dimension $\xi_{n}$. By code constructions, they proved the following result.

Theorem 7.3 (Lagarias and Shor [67]). For all n,

$$
\xi_{n} \leq n-\sqrt{n} / 3 \text {. }
$$

In addition, we have $\xi_{8} \leq 6, \xi_{9} \leq 7$ and $\xi_{10} \leq 7$. The last case was discovered by Lagarias and Shor [67], and the other cases were consequences of Mackey [73]. In general we have

$$
\xi_{n+1} \leq \xi_{n}+1 .
$$

This can be easily proved by a "stacking" construction that produces an $(n+1)$ dimensional tiling from an $n$-dimensional one, consisting of layers of $n$-dimensional tilings with successive layers shifted relative to each other to preclude any common faces between cubes in adjacent layers. However, we do not know the answer to the following problem. 
Problem 7.1 (Lagarias and Shor [67]). What is the order of $\xi_{n}$ as $n \rightarrow \infty$ ? Does $\xi_{n+1} \geq \xi_{n}$ hold for all $n$ ?

It is known (the Venkov-McMullen theorem (see [116])) that every convex translative tile is a lattice tile. On the other hand, Stein [99] and Szabó [102] discovered that, when $n \geq 5$, there is a star tile which is not a lattice star tile. In fact, by Theorem 6.1 and Theorem 7.2 one can construct such star tiles easily.

A family of unit cubes $I^{n}+Y$ will be called a $k$-fold tiling of $E^{n}$ if every point $\mathbf{x} \notin \partial\left(I^{n}\right)+Y$ lies in exactly $k$ cubes. Clearly a tiling is a 1 -fold tiling. To generalize Minkowski's conjecture to $k$-fold lattice tilings, Furtwängler [35] made the following ambitious conjecture in 1936 and proved it for $n \leq 3$.

Furtwängler's conjecture. Every $k$-fold lattice tiling $I^{n}+\Lambda$ of $E^{n}$ has a twin.

When G. Hajós did prove Minkowski's conjecture, unaware of Furtwängler's work, he studied this generalization again and restated it in the following version.

Furtwängler's conjecture in algebraic version. If each element $g$ of a finite abelian group $G$ is expressible in exact $k$ distinct ways as a product of elements coming from the cyclic subsets $A_{1}, A_{2}, \cdots, A_{n}$ respectively, where $A_{i}=$ $\left\{\mathbf{1}, \mathbf{g}_{i}, \cdots \mathbf{g}_{i}^{q_{i}-1}\right\}$, then $\mathbf{g}_{i}^{q_{i}}=\mathbf{1}$ for some $i, 1 \leq i \leq n$.

In the same paper, besides showing the $n \leq 3$ cases, Hajós disproved this statement for $n \geq 4$. In fact he did construct a 9-fold lattice tiling $I^{4}+\Lambda$ of $E^{4}$ which has no twin. Thus we have the following theorem about Furtwängler's conjecture.

Theorem 7.4 (Furtwängler [35] and Hajós [46]). When $n \leq 3$, every $k$-fold lattice tiling $I^{n}+\Lambda$ of $E^{n}$ has a twin; When $n \geq 4$, for some positive integer $k$ there is a $k$-fold lattice tiling $I^{n}+\Lambda$ of $E^{n}$ which has no twin.

By a comprehensive study of the algebraic version, in 1979 R.M. Robinson was able to determine all the integer pairs $\{n, k\}$ such that Furtwängler's conjecture is false. Thus Furtwängler's conjecture has been completely solved.

Theorem 7.5 (Robinson [90). There is a $k$-fold lattice tiling $I^{n}+\Lambda$ of $E^{n}$ which has no twin if and only if

1. $n=4$ and $k$ is a multiple of a square of an odd prime.

2. $n=5$ and $k=3$ or $k \geq 5$.

3. $n \geq 6$ and $k \geq 2$.

Both Theorem 7.4 and Theorem 7.5 were proved by studying equations in the group ring $\Re(G)$. For example, the condition of Furtwängler's conjecture can be rewritten as

$$
\widetilde{A_{1}} \widetilde{A_{2}} \cdots \widetilde{A_{n}}=k \widetilde{G}
$$

where

$$
\widetilde{X}=\sum_{\mathbf{g} \in X} \mathbf{g} .
$$

Remark 7.1. It was proved by Robinson 90 that every multiple translative tiling $I^{2}+X$ of $E^{2}$ has a twin and there is a 25-fold translative tiling $I^{3}+X$ of $E^{3}$ that has no twin. In 1982 Szabó [103] discovered that there is a 2-fold translative tiling $I^{n}+X$ of $E^{n}$ that has no twin whenever $n \geq 3$. 
Remark 7.2. The algebraic method is the key not only to solve Minkowski's conjecture, Keller's conjecture and Furtwängler's conjecture but also to deal with several other geometric problems about tiling. We refer the interested readers to Stein [100], Szabó [105] and Stein and Szabó [101].

\section{Miscellaneous}

In this section we will discuss some characterizations for parallelotopes, the closest relatives of the unit cubes. Since the topics of this section are not much related, we divide this section into three subsections.

8.1. A conjecture of Erdös and a problem of Klee. For convenience we will say that a subset $X$ of $E^{n}$ has property $\mathcal{E}$ if all the angles determined by the triples of points of $X$ are less than or equal to $\pi / 2$ and will say that it has property $\mathcal{K}$ if, for any pair of points $\{\mathbf{x}, \mathbf{y}\}$ of $X$, there are two parallel hyperplanes $H_{1}$ and $H_{2}$ such that $X$ is between $H_{1}$ and $H_{2}$. Clearly the $\mathcal{E}$ property implies the $\mathcal{K}$ property. In 1948 P. Erdös made a conjecture that

$$
\operatorname{card}\{X\} \leq 2^{n}
$$

holds for all $n$-dimensional sets with the $\mathcal{E}$ property. Similarly, in $1960 \mathrm{~V}$. Klee raised a problem to determine the value of $\max \{\operatorname{card}\{X\}\}$, where the maximum is over all $n$-dimensional sets with the $\mathcal{K}$ property. In 1962 L. Danzer and B. Grünbaum proved the following result which solves both Erdös' conjecture and Klee's problem.

Theorem 8.1 (Danzer and Grünbaum [25]). For all $n$-dimensional sets $X$ with the $\mathcal{K}$ property we have

$$
\operatorname{card}\{X\} \leq 2^{n},
$$

where the equality holds if and only if $X$ is the vertex set of a parallelotope; for all $n$-dimensional sets $Y$ with the $\mathcal{E}$ property we have

$$
\operatorname{card}\{Y\} \leq 2^{n},
$$

where the equality holds if and only if $Y$ is the vertex set of a rectangular parallelotope.

It is routine to show that $X$ is an $n$-dimensional set with the $\mathcal{K}$ property is equivalent to $\operatorname{conv}\{X\}-X$ is a finite packing. On the other hand, it was known even to Minkowski that, for any convex body $K, K+Y$ is a packing if and only if $\frac{1}{2}(K-K)+Y$ is a packing. Of course $K-K$ is always centrally symmetric. Therefore Klee's problem can be restated as, for an $n$-dimensional centrally symmetric convex body $C$, how many nonoverlapping translates of $C$ can have one common point? For convenience, we write the maximal number of such translates as $t(C)$. It is easy to see that all these translates are contained in $2 C+\mathbf{p}$, where $\mathbf{p}$ is the common point. Thus by volume estimation one can deduce that

$$
t(C) \leq 2^{n}
$$

and the equality holds if and only if $C$ is a parallelotope. In this way the theorem can be proved (see Aigner and Ziegler [4]). 
8.2. Inscribed and circumscribed ellipsoids. Let $C$ be a fixed $n$-dimensional centrally symmetric convex body centered at $\mathbf{o}$. For each ellipsoid $E$ centered at $\mathbf{o}$ there are a largest number $r(E)$ and a smallest number $r^{\prime}(E)$ such that

$$
r(E) \cdot E \subseteq C \subseteq r^{\prime}(E) \cdot E .
$$

Then we define

$$
\lambda(C)=\min \frac{r^{\prime}(E)}{r(E)},
$$

where the minimum is over all ellipsoids. Clearly we have $\lambda(C) \geq 1$, and the equality holds if and only if $C$ itself is an ellipsoid. As a counterpart, we have the following characterization for a parallelotope.

Theorem 8.2 (John [58] and Leichtweiß [71]). For all n-dimensional centrally symmetric convex bodies $C$ we have

$$
\lambda(C) \leq \sqrt{n},
$$

where the equality holds if and only if $C$ is a parallelotope.

Leichtweiß' key idea to prove this result is the following lemma, which can be verified easily.

Lemma 8.1 (Leichtweiß 71]). If $\alpha$ and $\beta$ are fixed numbers satisfying $|\alpha| \leq 1$ and $|\beta| \leq 1$, then among all the ellipsoids

$$
E_{\lambda}: \quad \sum_{i=1}^{n} x_{i}^{2}+\lambda\left(x_{1}-\alpha\right)\left(x_{1}+\beta\right) \leq 1
$$

for $0 \leq \lambda<\infty$ the unit ball has the smallest volume if and only if $\alpha \beta \geq 1 / n$.

Without loss of generality, we assume that the unit ball is the smallest circumscribed ellipsoid for $C$. Let $H_{1}=\left\{\mathbf{x}: x_{1}=\alpha\right\}$ and $H_{2}=\left\{\mathbf{x}: x_{2}=\beta\right\}$ be two supporting hyperplanes for $C$. It is easy to see that $|\alpha| \leq 1,|\beta| \leq 1$ and for any $\lambda \geq 0$, the ellipsoid

$$
\sum_{i=1}^{n} x_{i}^{2}+\lambda\left(x_{1}-\alpha\right)\left(x_{1}+\beta\right) \leq 1
$$

contains $C$. Based on this observation the inequality part of the theorem follows easily. Of course the characteristic part needs a more complicated argument.

Restricted to the ball and the cube, we have another simple sounding problem. Let $P$ be an $n$-dimensional polytope with $2 n$ facets and circumscribing the unit ball. We define

$$
\sigma(P)=\max \{\|\mathbf{x}\|: \mathbf{x} \in P\} .
$$

In 1994 C. Zong proposed the following problem.

Problem 8.1. Is it true that $\sigma(P) \geq \sqrt{n}$ and the equality holds if and only if $P$ is a cube?

By Euler's equation and the Dehn-Sommerville equations, Dalla, Larman, ManiLevitska and Zong [24] were able to prove the cases $n \leq 4$. So far the higherdimensional cases are still open. 
8.3. A conjecture of Hadwiger. To end this section and the whole article, we introduce a conjecture of Hadwiger. Let $K$ be an $n$-dimensional convex body and let $h(K)$ denote the smallest number of translates of int $(K)$ such that their union can cover $K$. In $1957 \mathrm{H}$. Hadwiger made the following conjecture.

Conjecture 8.1 (Hadwiger 43 ). For every $n$-dimensional convex body $K$ we have

$$
h(K) \leq 2^{n},
$$

where the equality holds if and only if $K$ is a parallelotope.

If $K$ is an $n$-dimensional parallelotope, then any translate of int $(K)$ cannot cover two of the $2^{n}$ vertices of $K$. Thus one can deduce $h(K)=2^{n}$ for this particular case. There are a great number of papers on this problem. However, so far this conjecture is open for $n \geq 3$. Since the known partial results are irrelevant to the characterization case, we will not list them here.

\section{ACKNOWLEDGEMENTS}

Part of this work was done while I was a visitor at IHES and MSRI. I am very grateful to these institutions for the support. For the helpful information, comments and suggestions I am obliged to G.M. Ziegler, J.C. Lagarias, M. Henk, P.M. Gruber, R. Graham, J.E. Goodman and R.J. Gardner.

\section{REFERENCES}

1. F. Affentranger and R. Schneider, Random projections of regular simplices, Discrete Comput. Geom. 7 (1992), 219-226. MR1149653 (92k:52008)

2. S.S. Agaian, Hadamard Matrices and Their Applications, Springer-Verlag, Berlin, 1985. MR.0818740 (87k:05038)

3. O. Aichholzer, Extremal properties of $0 / 1$ polytopes of dimension 5, PolytopesCombinatorics and Computation, DMV Sem. 29 (2000), 111-130. MR1785295 (2002k:52013)

4. M. Aigner and G.M. Ziegler, Proofs from the Book (third edition), Springer-Verlag, Berlin, 2004. MR2014872 (2004h:00002)

5. K. Ball, Cube slicing in $R^{n}$, Proc. Amer. Math. Soc. 97 (1986), 465-473. MR0840631 (87g:60018)

6. K. Ball, Logarithmically concave functions and sections of convex sets in $R^{n}$, Studia Math. 88 (1988), 69-84. MR0932007 (89e:52002)

7. K. Ball, Volumes of sections of cubes and related problems, Lecture Notes in Math. 1376 (1989), 251-260. MR1008726 (90i:52019)

8. K. Ball, An elementary introduction to modern convex geometry, Flavors of Geometry (edited by S. Levy), Cambridge University Press, Cambridge, 1997. MF,1491097 (99f:52002)

9. I. Bárány and L. Lovász, Borsuk's theorem and the number of facets of centrally symmetric polytopes, Acta Math. Sci. Hungar. 40 (1982), 323-329. MR0686332 (84g:52007)

10. I. Bárány and A. Pór, On 0-1 polytopes with many facets, Adv. Math. 161 (2001), 209-228. MR 1851645 (2003e:52013)

11. G. Barba, Intorno al teorema di Hadamard sui determinanti a valore massimo, Giorn. Mat. Battaglia 71 (1933), 70-86.

12. A. Below, U. Brehm, J.A. De Loera and J. Richter-Gebert, Minimal simplicial dissections and triangulations of convex 3-polytopes, Discrete Comput. Geom. 24 (2000), 35-48. MR 1765232 (2001g:52021)

13. L.J. Billera, R. Cushman and J.A. Sanders, The Stanley decomposition of the harmonic oscillator, Nederl. Akad. Wetensch. Indag. Math. 50 (1988), 375-393. MR0976522 (89m:13013)

14. L.J. Billera and A. Sarangarajan, All 0-1 polytopes are traveling salesman polytopes, Combinatorica 16 (1996), 175-188. MR:1401891 (97j:52016) 
15. C. Borell, Convex set functions in d-space, Period. Math. Hungar. 6 (1975), 111-136. MR 0404559 (53:8359)

16. K. Böröczky Jr. and M. Henk, Random projections of regular polytopes, Arch. Math. (Basel) 73 (1999), 465-473. MR 1725183 (2001b:52004)

17. H.J. Brascamp and E.H. Lieb, Best constants in Young's inequality, its converse, and its generalization to more than three functions, Adv. in Math. 20 (1976), 151-173. MR0412366 $(54: 492)$

18. J. Brenner and L. Cummings, The Hadamard maximum determinant problem, Amer. Math. Monthly 79 (1972), 626-629. MF 0301030(46:190)

19. M.N. Broadie and R.W. Cottle, A note on triangulating the 5-cube, Discrete Math. 52 (1984), 39-49. MR0765283 (86c:52011)

20. G.D. Chakerian and P. Filliman, The measures of the projections of a cube, Studia Sci. Math. Hungar. 21 (1986), 103-110. MR0898848 (88f:52007)

21. G.F. Clements and B. Lindström, A sequence of $( \pm 1)$-determinants with large values, Proc. Amer. Math. Soc. 16 (1965), 548-550. MR0178001(31:2259)

22. K. Corrádi and S. Szabó, A combinatorial approach for Keller's conjecture, Period. Math. Hungar. 21 (1990), 95-100. MR.1070948(91j:52025)

23. R.W. Cottle, Minimal triangulations of the 4-cube, Discrete Math. 40 (1982), 25-29. MR 0676709 (84d:05065a)

24. L. Dalla, D.G. Larman, P. Mani-Levitska and C. Zong, The blocking numbers of convex bodies, Discrete Comput. Geom. 24 (2000), 267-277. MF.1758049(2001d:52011)

25. L. Danzer and B. Grünbaum, Über zwei Probleme bezüglich konvexer Körper von P. Erdös und von V.L. Klee, Math. Z. 79 (1962), 95-99. MR0138040 (25:1488)

26. P. Delsarte, Bounds for unrestricted codes, by linear programming, Philips Research Reports 27 (1972), 272-289. MR0314545 (47:3096)

27. P. Delsarte, An algebraic approach to the association schemes of coding theory, Philips Research Reports, Suppl. 10 (1973), 1-97. MR0384310 (52:5187)

28. A. Dvoretzky, Some results on convex bodies and Banach spaces, Proc. Internat. Symp. Linear Spaces (1961), 123-160. MR0139079 (25:2518)

29. A. Dvoretzky, Some near-sphericity results, Proc. Symp. Pure Math. 7 (1963), 203-210. MR $0158308(28: 1533)$

30. M.E. Dyer, Z. Füredi and C. McDiarmid, Volumes spanned by random points in the hypercube, Random Structures Algorithms 3 (1992), 91-106. MR1139489 (92j:52010)

31. H. Ehlich, Determinantenabschätzungen für binäre Matrizen, Math. Z. 83 (1964), 123-132. MR0160792 (28:4003)

32. H. Ehlich, Determinantenabschätzungen für binäre Matrizen mit $n \equiv 3 \bmod 4$, Math. Z. 84 (1964), 438-447. MR0168573 (29:5833)

33. L. Fejes Tóth, Regular Figures, Pergamon Press, London, 1964. MR0165423 (29:2705)

34. T. Fleiner, V. Kaibel and G. Rote, Upper bounds on the maximal number of facets of $0 / 1$ polytopes, European J. Combin. 21 (2000), 121-130. MR1737332 (2001d:52019)

35. P. Furtwängler, Über Gitter konstanter Dichte, Monatsh. Math. Phys. 43 (1936), 281-288.

36. E.N. Gilbert, A comparison of signaling alphabets, Bell System Tech. J. 31 (1952), 504-522.

37. N.A. Grigorév, Regular simplices inscribed in a cube and Hadamard matrices, Proc. Steklov Inst. Math. 152 (1982), 97-98. MF0603815 (82d:10043)

38. P. Gritzmann, V. Klee and D.G. Larman, Largest $j$-simplices in $n$-polytopes, Discrete Comput. Geom. 13 (1995), 477-515. MR.1318791 (96b:52019)

39. P.M. Gruber, Zur Charakterisierung konvexer Körper. Über einen Satz von Rogers und Shephard. II. Math. Ann. 184 (1970), 79-105. MR0256266 (41:922)

40. W. Gruner, Einlagerung des regulären $n$-Simplex in den $n$-dimensionalen Würfel, Comment. Math. Helvetici 12 (1939/40), 149-152. MR0001205(1:195c)

41. U. Haagerup and H.J. Munkholm, Simplices of maximal volume in hyperbolic $n$-space, Acta Math. 147 (1981), 1-12. MR0631085 (82j:53116)

42. J. Hadamard, Résolution d'une question relativ aux déterminants, Bull. Sci. Math. 28 (1893), 240-246.

43. H. Hadwiger, Ungelöste Probleme Nr. 20, Elem. Math. 12 (1957), 121.

44. H. Hadwiger, Gitterperiodische Punktmengen und Isoperimetrie, Monatsh. Math. 76 (1972), 410-418. MR0324550 (48:2902) 
45. M. Haiman, A simple and relatively efficient triangulation of the $n$-cube, Discrete \& Comput. Geom. 6 (1991), 287-289. MR1098809 (92e:52011)

46. G. Hajós, Über einfache und mehrfache Bedeckung des $n$-dimensionalen Raumes mit einem Würfelgitter, Math. Z. 47 (1942), 427-467. MR0006425 (3:302b)

47. G. Hajós, Sur la factorisation des groupes abelians, Ćasopis. Pést. Mat. Fys. 74 (1950), 157-162. MR0045727|(13:623a)

48. P. Hall, Jr., Combinatorial Theory, Blaisdell, Waltham, Mass., 1966.

49. D. Hensley, Slicing the cube in $R^{n}$ and probability, Proc. Amer. Math. Soc. 73 (1979), 95-100. MR0512066 (80b:60025)

50. D. Hensley, Slicing convex bodies-Bounds for slice area in terms of the body's covariance, Proc. Amer. Math. Soc. 79 (1980), 619-625. MR0572315 (81j:52008)

51. M. Hudelson, V. Klee and D.G. Larman, Largest $j$-simplices in $d$-cubes: Some relatives of the Hadamard maximum determinant problem, Linear Algebra Appl. 241-243 (1996), 519-598. MR 1400454 (98c:15025)

52. R.B. Hughes and M.R. Anderson, A triangulation of the 6-cube with 308 simplices, Discrete Math. 117 (1993), 253-256. MR1226146 (94b:05059)

53. R.B. Hughes, Minimum-cardinality triangulations of the $d$-cube for $d=5$ and $d=6$, Discrete Math. 118 (1993), 75-118. MR1230056 (94h:52025)

54. R.B. Hughes, Lower bounds on cube simplexity, Discrete Math. 133 (1994), 123-138. MF, 1298968 (95i:90033)

55. R.B. Hughes and M.R. Anderson, Simplexity of the cube, Discrete Math. 158 (1996), 99150. MR:1411113 (97g:90083)

56. D.B. Jaffe, Optimal binary linear codes of length $\leq 30$, Discrete Math. 223 (2000), 135-155. MF1782044 (2001i:94073)

57. H. Jansen, Lückenlose Ausfüllung des $R_{n}$ mit gitterförmig angeordneten $n$-dimensionalen Quadern, Dissertation, Kiel, 1909.

58. F. John, Extremum problems with inequalities as subsidiary conditions, Courant Anniv. Vol. (1948), 187-204. MF0030135 (10:719b)

59. J. Kahn, J. Komlós and E. Szemerédi, On the probability that a random \pm 1 -matrix is singular, J. Amer. Math. Soc. 8 (1995), 223-240. MR.1260107 (95c:15047)

60. M. Kanter, Unimodality and dominance for symmetric random vectors, Trans. Amer. Math. Soc. 229 (1977), 65-85. MR0445580 (56:3917)

61. O.H. Keller, Über die lückenlose Einfüllung des Raumes mit Würfeln, J. reine angew. Math. 163 (1930), 231-248.

62. O.H. Keller, Ein Satz über die lückenlose Erfüllung des 5- und 6-dimensional Raumes mit Würfeln, J. reine angew. Math. 177 (1937), 61-64.

63. M.N. Kolountzakis, Lattice tilings by cubes: whole, notched and extended, Electron. J. Combin. 5 (1998), 1-11. MR1605065 (98k:52054)

64. U.H. Kortenkamp, J. Richter-Gebert, A. Sarangarajan and G.M. Ziegler, Extremal properties of 0/1-polytopes, Discrete Comput. Geom. 17 (1997), 439-448. MR 1455692 (98d:52016)

65. G. Kowalewski, Einführung in der Determinantentheorie einschliesslich der Fredholmschen Determinanten, Walter de Gruyter \& Co., Berlin, 1954. MR0064003 (16:210b)

66. J.C. Lagarias and P.W. Shor, Keller's cube-tiling conjecture is false in high dimensions, Bull. Amer. Math. Soc. 27 (1992), 279-283. MR1155280 (93e:52040)

67. J.C. Lagarias and P.W. Shor, Cube-tilings of $R^{n}$ and nonlinear codes, Discrete Comput. Geom. 11 (1994), 359-391. MR.1273224(95e:52044)

68. D.G. Larman and P. Mani, Almost ellipsoidal sections and projections of convex bodies, Proc. Cambridge Philos. Soc. 77 (1975), 529-546. MF 0377697 (51:13866)

69. C. Lee, Triangulating the $d$-cube, Discrete Geometry and Convexity (J.E. Goodman, E. Lutwak, J. Malkevitch and R. Pollack, eds.), New York Academy of Sciences, New York (1985), 205-211. MR0809208 (87d:52011)

70. C. Lee, Subdivisions and triangulations of polytopes, Handbook of Discrete and Computational Geometry, CRC Press, Boca Raton (1997), 271-290. MR1730170

71. K. Leichtweiß, Über die affine Exzentrizität konvexer Körper, Arch. Math. 10 (1959), 187199. MR0107843 (21:6565)

72. Y. Lonke, On random sections of the cube, Discrete Comput. Geom. 23 (2000), 157-169. MF.1739603 (2001e:60022) 
73. J. Mackey, A cube tiling of dimension eight with no facesharing, Discrete 8 Comput. Geom. 28 (2002), 275-279. MR.1920144 (2003g:52041)

74. P.S. Mara, Triangulations for the cube, J. Combin. Theory (A), 20 (1976), 170-177. MR 0406838 (53:10624)

75. R.J. McEliece, E.R. Rodemich, H. Rumsey Jr., and L. R. Welch, New upper bounds on the rate of a code via the Delsarte-Macwilliams inequalities, IEEE Trans. Inform. Theory $\mathbf{2 3}$ (1977), 157-166. MR0439403 (55:12296)

76. P. McMullen, Volumes of projections of unit cubes, Bull. London Math. Soc. 16 (1984), 278-280. MR0738519|(85j:52019)

77. A.I. Medyanik, A regular simplex inscribed in a cube, half-circulant Hadamard matrices, and Gaussian sums, Mat. Fiz. Anal. Geom. 8 (2001), 58-81. MF.1846358(2002h:05029)

78. H. Minkowski, Geometrie der Zahlen, Teubner, Leipzig, 1896.

79. H. Minkowski, Diophantische Approximationen, Teubner, Leipzig, 1907.

80. M.G. Neubauer and A.J. Radcliffe, The maximum determinant of \pm 1 matrices, Linear Algebra Appl. 257 (1997), 289-306. MR1441716 (98a:15014)

81. M.G. Neubauer, W. Watkins and J. Zeitlin, Maximal $j$-simplices in the real $d$-dimensional unit cube, J. Combin. Theory (A) 80 (1997), 1-12. MF,1472104 (98j:52018)

82. D. Orden and F. Santos, Asymptotically efficient triangulations of the $d$-cube, Discrete Comput. Geom. 30 (2003), 509-528. MR2013970 (2004i:68231)

83. M.I. Ostrovskii, Minimal-volume shadows of cubes, J. Funct. Anal. 176 (2000), 317-330. MR.1784418 (2002a:46004)

84. R.E.A.C, Paley, On orthogonal matrices, J. Math. Phys. 12 (1933), 311-320.

85. O. Perron, Über lückenlose Ausfüllung des $n$-dimensionalen Raumes durch kongruente Würfel I; II, Math. Z. (1940), 1-26; 161-180. MR0003041 (2:153e); MR0002185 (2:11d)

86. O. Perron, Modulartige lückenlose Ausfüllung des $R^{n}$ mit kongruenten Würfeln I; II, Math. Ann. 117 (1940), 415-447; 117 (1941), 609-658. MR0003042|(2:153f) MR0006068(3:253a)

87. V.S. Pless, W.C. Huffman and R.A. Brualdi (eds.), Handbook of Coding Theory, Elsevier, 1998. MR 1667936 (2000h:94001)

88. A. Prékopa, On logarithmically concave measures and functions, Acta Sci. Math. (Szeged) 34 (1973), 335-343. MR0404557 (53:8357)

89. L. Rédei, Die neue Theorie der endlichen abelschen Gruppen und Verallgemeinerung des Hauptsatzes von Hajós, Acta Math. Acad. Sci. Hungar. 16 (1965), 327-373. MR0186729 $(32: 4187)$

90. R.M. Robinson, Multiple tiling of $n$-dimensional space by unit cubes, Math. Z. 166 (1979), 225-264. MR0526466 (80g:05027)

91. J.F. Sallee, A triangulation of the $n$-cube, Discrete Math. 40 (1982), 81-86. MR0676714 (84d:05065b)

92. J.F. Sallee, A note on minimal triangulations of an n-cube, Discrete Applied Math. 4 (1982), 211-215. MR0675850 (84g:52019)

93. J.F. Sallee, Middle-cut triangulations of the n-cube, SIAM J. Algbraic Discrete Methods 5 (1984), 407-419. MR0752044 (86c:05054)

94. K.W. Schmidt, Lower bounds for maximal (0,1)-determinants, SIAM J. Appl. Math. 19 (1970), 440-441. MR0265382 (42:292)

95. T. Schmidt, Über die Zerlegung des $n$-dimensionalen Raumes in gitterförmig angeordnete Würfeln, Schr. Math. Semin. U. Institut angew. Math. Univ. Berlin 1 (1933), 186-212.

96. G.C. Shephard, Combinatorial properties of associated zonotopes, Canad. J. Math. 26 (1974), 302-321. MR0362054 (50:14496)

97. N.J.A. Sloane, Error-correcting codes and invariant theory: new applications of a nineteenth-century technique, Amer. Math. Monthly 84 (1977), 82-107. MR0424398 $(54: 12361)$

98. W.D. Smith, A lower bound for the simplexity of the $n$-cube via hyperbolic volumes, European J. Combin. 21 (2000), 131-137. MR1737333 (2001c:52004)

99. S.K. Stein, A symmetric star body that tiles but not as a lattice, Proc. Amer. Math. Soc. 36 (1972), 543-548. MR0319058 (47:7604)

100. S.K. Stein, Algebraic tiling, Amer. Math. Monthly 81 (1974), 445-462. MR0340063 (49:4819)

101. S.K. Stein and S. Szabó, Algebra and Tiling, Mathematical Association of America, 1994. MR.1311249(95k:52035) 
102. S. Szabó, On mosaics consisting of multidimensional crosses, Acta Math. Acad. Sci. Hungar. 38 (1981), 191-203. MR0634580 (83a:51021)

103. S. Szabó, Multiple tilings by cubes with no shared faces, Aequationes Math. 25 (1982), 83-89. MR0716380 (85c:52024)

104. S. Szabó, A reduction of Keller's conjecture, Period. Math. Hungar. 17 (1986), 265-277. MR.0866636 (88j:52032)

105. S. Szabó, Cube tilings as contributions of algebra to geometry, Beiträge Algebra Geom. 34 (1993), 63-75. MR.1239279 (94f:52039)

106. W.P. Thurston, The Geometry and Topology of 3-manifolds, Lecture Notes from Princeton University, 1977.

107. J.D. Vaaler, A geometric inequality with applications to linear forms, Pacific J. Math. 83 (1979), 543-553. MR0557952 (81d:52007)

108. J.H. van Lint, Introduction to Coding Theory, Springer-Verlag, New York, 1982. MR.0658134(84e:94001)

109. R.R. Varshamov, Estimate of the number of signals in error correcting codes, Dokl. Akad. Nauk SSSR 117 (1957), 739-741.

110. J. Williamson, Determinants whose elements are 0 and 1, Amer. Math. Monthly 53 (1946), 427-434. MR0017261 (8:128g)

111. M. Wojtas, On Hadamard's inequality for the determinants of order non-divisible by 4, Colloq. Math. 12 (1964), 73-83. MR0168574(29:5834)

112. M. Yamada, Some new series of Hadamard matrices, J. Austral. Math. Soc. 46 (1989), 371-383. MR 0987555 (90e:05022)

113. C.H. Yang, Some designs for maximal $(+1,-1)$-determinant of order $n \equiv 2(\bmod 4)$, Math. Comp. 20 (1966), 147-148; 22 (1968), 174-180; 23 (1969), 201-205. MR0188093 (32:5534)

114. C.H. Yang, A construction for maximal $(+1,-1)$-matrix of order 54, Bull. Amer. Math. Soc. 72 (1966), 293. MR0188239 (32:5678)

115. G.M. Ziegler, Lectures on 0/1-Polytopes, Polytopes-Combinatorics and Computation, DMV Sem. 29 (2000), 1-41. MR,1785291 (2001e:52017)

116. C. Zong, Strange Phenomena in Convex and Discrete Geometry, Springer-Verlag, New York, 1996. MR:1416567 (97m:52001)

117. C. Zong, The Cube - A Window to Convex and Discrete Geometry, Cambridge University Press, in press.

School of Mathematical Sciences, Peking University, Beijing 100871, People's RePUBLIC OF CHINA

E-mail address: cmzong@math.pku.edu.cn 